\title{
Generalized polarized manifolds with low second class
}

\author{
Antonio LANTERI and Andrea Luigi TIRONI \\ (Received July 27, 2020) \\ (Revised June 18, 2021)
}

\begin{abstract}
On a smooth complex projective variety $X$ of dimension $n$, consider an ample vector bundle $\mathscr{E}$ of rank $r \leq n-2$ and an ample line bundle $H$. A numerical character $m_{2}=m_{2}(X, \mathscr{E}, H)$ of the triplet $(X, \mathscr{E}, H)$ is defined, extending the well-known second class of a polarized manifold $(X, H)$, when either $n=2$ or $H$ is very ample. Under some additional assumptions on $\mathscr{F}:=\mathscr{E} \oplus H^{\oplus(n-r-2)}$, triplets $(X, \mathscr{E}, H)$ as above whose $m_{2}$ is small with respect to the invariants $d:=c_{n-2}(\mathscr{F}) H^{2}$ and $g:=1+\frac{1}{2}\left(K_{X}+\right.$ $\left.c_{1}(\mathscr{F})+H\right) \cdot c_{n-2}(\mathscr{F}) \cdot H$ are studied and classified.
\end{abstract}

\section{Introduction}

Let $S$ be a smooth complex projective surface embedded by a very ample line bundle $L$. Identify $S$ with its image in $\mathbb{P}^{N}, N=\operatorname{dim} H^{0}(S, L)-1$, via the embedding associated with $L$ and think of the linear system $|L|$ corresponding to the elements of $H^{0}(S, L)$ as the hyperplane linear system of $S$. Consider also the dual variety $\mathscr{D}(S)$ of $S$, i.e. the subset of $|L|$ parameterizing the tangent hyperplanes. If $(S, L) \neq\left(\mathbb{P}^{2}, \mathcal{O}_{\mathbb{P}^{2}}(1)\right)$, then $\mathscr{D}(S)$ is a hypersurface in the dual projective space $\mathbb{P}^{N \vee}$ (identified with $|L|$ ), and its degree $m$ is usually called the class of $S$. More generally, for a projective manifold $X \subset \mathbb{P}^{N}$ one can consider its second class $m_{2}$, namely the class of its general surface section, which is always positive, unless $X$ is a linear space, by what we said. Like for the degree and the sectional genus, the study of $m_{2}$ contributed to a large literature on the classification of smooth projective varieties with small invariants. In particular, it is known that for $m \leq 29, S$ is a ruled surface and pairs $(S, L)$ occurring for $m \leq 25$ are classified (see [16, p. 195], and [34, Prop. 3.2]). Moreover, for $m \leq 11$ only $\left(\mathbb{P}^{2}, \mathcal{O}_{\mathbb{P}^{2}}(e)\right), e=1,2$, and scrolls may occur (e.g. see Remark 1).

The first author is a member of G.N.S.A.G.A. of the Italian INdAM. He would like to thank the Projects PRIN 2010-11 and 2015 Geometry of Algebraic Varieties and the University of Milano for partial support.

The second author was partially supported by the National Project Anillo ACT 1415 PIA CONICYT and the Proyecto VRID N.219.015.023-INV of the University of Concepción.

2010 Mathematics Subject Classification. Primary 14F05; Secondary 14J60, 14J25, 14N30.

Key words and phrases. Ample vector bundle; special variety; surface. 
Due to the fact that $m=c_{2}\left(J_{1}(L)\right)$, the second Chern class of the first jet bundle of $L$, in recent years the study of small values of $m$ for embedded surfaces has been reconsidered and transplanted in the wider setting of ample line bundles. In particular, Palleschi and Turrini ([30]) started to classify polarized surfaces $(S, H)$ when $H$ is only assumed to be ample on $S$ by studying small values of $c_{2}\left(J_{1}(H)\right)$ and of $c_{2}\left(J_{1}(H)\right)-d$, where $d=H^{2}$, in line with classical papers by Marchionna [29] and Gallarati [13], [14]. For pairs $(S, H)$ as above the situation is different from the classical case because already for $c_{2}\left(J_{1}(H)\right)=5$ a nonruled surface occurs. Sometimes, in this context, $m:=c_{2}\left(J_{1}(H)\right)$ is referred to as the generalized class of the polarized surface $(S, H)$.

The aim of this paper is twice. First of all, starting from Table 1, which combines the list in [30, Theorem 4.3] with results of Fujita [6] and Yokoyama [35], summarizing what is known for polarized surfaces with $m-d \leq 8$, we prove new results concerning the case $2 g+1 \leq m-d \leq 2 g+2$, where $g$ is the sectional genus (Theorem 1) and the case of polarized nonruled surfaces with $m-2 d<2 g$ (Proposition 1). Both will play a relevant role in the sequel. Next we revisit the study of the character $m$ in the framework of ample vector bundles. We generalize $m_{2}$ from a projective manifold $X$ polarized by a very ample line bundle $L$ to triplets $(X, \mathscr{E}, H)$ in an appropriate vector bundle setting, and we study the objects giving rise to small values of this character. Roughly speaking, on a smooth complex projective variety $X$ of dimension $n$, consider an ample vector bundle $\mathscr{E}$ of rank $r, 2 \leq r \leq n-2$, and an ample line bundle $H$. By considering the triplet $(X, \mathscr{E}, H)$ and the ample vector bundle of rank $n-2$ on $X$ given by $\mathscr{F}:=\mathscr{E} \oplus H^{\oplus(n-r-2)}$, we define the generalized class $m_{2}=m_{2}(X, \mathscr{E}, H)$ of $(X, \mathscr{E}, H)$ as

$$
m_{2}:=\left[c_{2}\left(\Omega_{X} \oplus \operatorname{det} \mathscr{F}\right)+c_{1}^{2}-c_{2}+H^{2}\right] \cdot c_{n-2}+4(g-1),
$$

where $c_{i}:=c_{i}(\mathscr{F})$ for $i=1,2, \ldots, n-2$, and $g:=1+\frac{1}{2}\left(K_{X}+c_{1}+H\right) \cdot H$. $c_{n-2}$. From now on we simply write $m_{2}$ for $m_{2}(X, \mathscr{E}, H)$.

If $\mathscr{F}$ admits a section vanishing on a smooth surface $S$, it turns out that $m_{2}=c_{2}\left(J_{1}\left(H_{S}\right)\right)$, the generalized class of the polarized surface $\left(S, H_{S}\right)$. Moreover, for $H$ very ample and $\mathscr{E}=H^{\oplus(n-2)}, m_{2}$ is just the second class of the projective manifold $X$ embedded in $\mathbb{P}^{N}$ via $|H|$.

This allows us to revisit and extend several classification results for surfaces of small class in the setting of ample vector bundles. Actually, under the above assumption on $\mathscr{F}$, we show that $m_{2} \geq d$, where $d:=c_{n-2}(\mathscr{F}) \cdot H^{2}$, except for $(X, \mathscr{E}, H)=\left(\mathbb{P}^{n}, \mathcal{O}_{\mathbb{P}^{n}}(1)^{\oplus r}, \mathcal{O}_{\mathbb{P}^{n}}(1)\right)$, or $\left(\mathbb{P}^{n}, \mathcal{O}_{\mathbb{P}^{n}}(1)^{\oplus(n-2)}, \mathcal{O}_{\mathbb{P}^{n}}(2)\right)$, and we describe completely the triplets satisfying equality (see Theorem 2).

Then by putting $\delta:=m_{2}-d$, in line with the classical case, we study small positive values of $\delta$ by proving that $\delta \geq 6$, apart from few triplets $(X, \mathscr{E}, H)$, 
which are precisely described (Theorem 3). As a consequence of these results, we describe the possible triplets $(X, \mathscr{E}, H)$ with $m_{2} \leq 6$. Moreover, we carry on our analysis to prove that if $m_{2}>6$, then $m_{2} \geq 10$, provided that $S$ has nonnegative Kodaira dimension. Including the sectional genus $g$ into the picture, we characterize triplets for which $\delta \leq 2 g+2$ (Proposition 4 and Theorem 4) and we show that $\delta \geq 2 g+d$ if $S$ has non-negative Kodaira dimension. Moreover, as expected, the stronger are the properties enjoyed by the line bundle $H_{S}$ (spannedness by global sections, very ampleness), the larger are the values of $m_{2}$ attained by our results. In particular, assuming that $H_{S}$ is spanned by global sections, we list the triplets with $m_{2} \leq 11$, those with $\delta \leq 2 g+2$, as well as those with $\delta \leq 2 g+5$ provided that $S$ has non-negative Kodaira dimension (Proposition 5 and Theorem 7). In connection with this, we have the opportunity to correct a mistake in [24] (see Remark 7 ii)). On the other hand, under the assumption that $H_{S}$ is very ample, we revisit the above results and finally we prove that $\delta \geq 2 g+11$ if $S$ has non-negative Kodaira dimension.

A great help in our analysis is provided by a number of results on ample vector bundles having a section which vanishes on a surface of some special kind ([5], [17], [19], [20], [21], [22]). The strategy is the following: first, looking at the difference $\delta$, which can be expressed in terms of geometric and topological characters, we show, extending or refining some known results, that the polarized surface $\left(S, H_{S}\right)$ must belong to a precise list of pairs. Next, by applying the results on ample vector bundles mentioned before we succeed to reduce (sometimes drastically) these lists to a very short number of cases, for which we obtain a rather complete description of $\mathscr{E}$ and $H$ according to the admissible structure of $X$. For example, in some instances $S$ could "a priori" be a minimal elliptic surface, whose elliptic fibration turns out to be endowed with some multiple fibers, but this possibility is ruled out by [22]. Therefore these cases do not lift to the vector bundle setting.

The paper is organized as follows. Section 2 contains miscellaneous preliminary results on polarized surfaces $(S, L)$ with special regard to pairs for which $c_{2}\left(J_{1}(L)\right)-L^{2}$ is small. In particular, in this setting, we prove new results concerning both ruled and nonruled surfaces. In Section 3 the invariant $m_{2}$ is introduced for triplets $(X, \mathscr{E}, H)$ in an appropriate setting and triplets for which $\delta$ is small are analyzed. Moreover, lists of triplets with low $m_{2}$ are derived from this study. In Section 4 significant bounds for $\delta$ expressed in terms of the sectional genus $g$ are discussed. Finally, in Section 5 all the above matter is reconsidered under the extra assumption that the line bundle $H_{S}$ is ample and spanned (Subsection 4.1) or even very ample (Subsection 4.2).

We work over the field of complex numbers and we use the standard notation and terminology from algebraic geometry. In particular, 


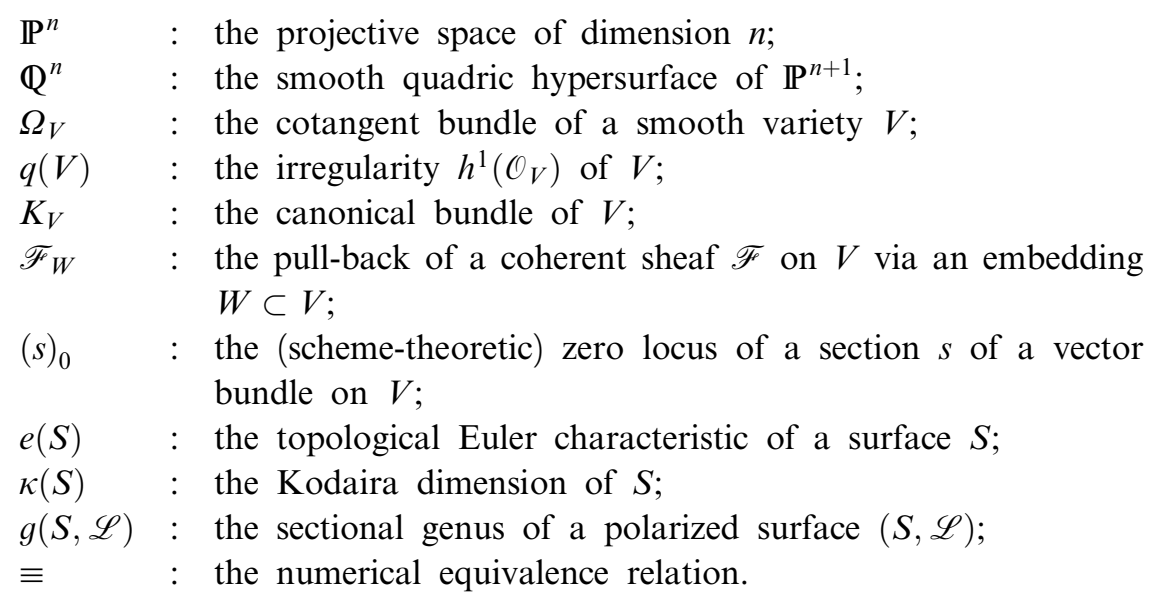

With a little abuse, we adopt the additive notation for the tensor product of line bundles. We say that a smooth surface $S$ is ruled if it is birationally ruled, i.e. if $\kappa(S)=-\infty ; S$ is said to be geometrically ruled if it is a $\mathbb{P}^{1}$-bundle over a smooth curve. To denote a geometrically ruled surface of invariant $e$ over a smooth curve of genus $q:=q(S)$ we use the non-standard symbol $S_{q, e}$ (in particular, $S_{0, e}$ is the Segre-Hirzebruch surface of invariant $e$ ); however, as usual (e.g., see [15, Chapter $\mathrm{V}, \S 2]) C_{0}$ and $f$ will stand for a section of minimal self-intersection $-e$ and a fiber, respectively. We recall that $e \geq-q$ (Nagata inequality).

\section{Polarized surfaces $(S, L)$ with small class}

Here are some general facts concerning polarized surfaces $(S, L)$. Apart from the interest in connection with the study of pairs for which the difference $m-d$ is small, they will be useful in the basic setting introduced in Section 3.

For the convenience of the reader, we sum up in Table 1 known results concerning polarized surfaces $(S, L)$ whose class $m:=c_{2}\left(J_{1}(L)\right)$ is small compared to the degree $d:=L^{2}$. We set $q:=q(S), g=g(S, L)$, and we denote by $\left(S^{\prime}, L^{\prime}\right)$ a minimalization of $(S, L)$, when $S$ is not minimal, as in [30]. Recall that letting $\eta: S \rightarrow S^{\prime}$ be the corresponding birational morphism, we have $L=$ $\eta^{*} L^{\prime}-\sum v_{i} E_{i}$, where $E_{i}, i=1, \ldots, s$, are the exceptional curves contracted by $\eta$ and $v_{i} \geq 1$ for every $i$.

In particular, note that all pairs $(S, L)$ with $m \leq 9$ are included in Table 1 . The basic source for Table 1 is [30, Section 4], taking into account some progress in the classification of polarized surfaces of sectional genus two, compared to [4]. Moreover, as to N. 11, we note that the description of $L$ provided in [4, Theorem 2.7, d)] has been improved by Fujita (see [8, Theorem 15.7] and 


\begin{tabular}{|c|c|c|c|c|c|c|c|c|}
\hline N. & $m-d$ & $d$ & $g$ & $q$ & $\kappa(S)$ & $e(S)$ & $(S, L)$ & $\left(S^{\prime}, L^{\prime}\right)$ \\
\hline 1 & -1 & 1,4 & 0 & 0 & $-\infty$ & 3 & $\left(\mathbb{P}^{2}, \mathcal{O}_{\mathbb{P}^{2}}(e)\right), e=1,2$ & - \\
\hline 2 & 0 & $\geq 1$ & $q$ & $\geq 0$ & $-\infty$ & $4-4 q$ & $\begin{array}{l}\text { scroll over a smooth curve of } \\
\text { genus } q\end{array}$ & - \\
\hline 3 & 3 & 9 & 1 & 0 & $-\infty$ & 3 & $\left(\mathbb{P}^{2}, \mathcal{O}_{\mathbb{P}^{2}}(3)\right)$ & - \\
\hline 4 & 4 & 8 & 1 & 0 & $-\infty$ & 4 & $\left(\mathbb{P}^{1} \times \mathbb{P}^{1}, \mathcal{O}_{\mathbb{P}^{1} \times \mathbb{P}^{1}}(2,2)\right)$ & - \\
\hline 5 & 4 & 8 & 1 & 0 & $-\infty$ & 4 & the blow-up at a point of & N. 3 \\
\hline 6 & 4 & 3 & 2 & 1 & $-\infty$ & 0 & $\left(S_{1,-1},\left[3 C_{0}-f\right]\right)$ & - \\
\hline 7 & 4 & 4 & 2 & 1 & $-\infty$ & 0 & $\begin{array}{c}\left(S_{1, e},\left[2 C_{0}+(e+1) f\right]\right) \\
e=-1,0\end{array}$ & - \\
\hline 8 & 4 & 1 & 2 & 1 & 1 & 0 & $\begin{array}{l}S \rightarrow \mathbb{P}^{1} \text { is a minimal elliptic } \\
\text { surface with multiple fibers }\end{array}$ & - \\
\hline 9 & 4 & 2 & 2 & 2 & 0 & 0 & $\begin{array}{l}S \text { is the Jacobian of a smooth } \\
\text { curve } C \text { of genus } 2, L \equiv C \\
\text { embedded in } S \text { and } h^{0}(L)=1\end{array}$ & - \\
\hline 10 & 4 & 2 & 2 & 2 & 0 & 0 & $\begin{array}{c}S \cong C_{1} \times C_{2}, C_{i} \text { is an elliptic } \\
\text { curve for } i=1,2, L \equiv C_{1}+C_{2} \\
\quad \text { and } h^{0}(L)=1\end{array}$ & - \\
\hline 11 & 4 & 2 & 2 & 1 & 0 & 0 & $\begin{array}{c}S \text { is a bielliptic surface, }|L|= \\
\{Z+F\}, Z \text { a section, } F \text { a fiber } \\
\text { of the Albanese fibration }\end{array}$ & - \\
\hline 12 & 5 & 7 & 1 & 0 & $-\infty$ & 5 & the blow-up at two points of & N. 3 \\
\hline 13 & 5 & 1 & 2 & 2 & 0 & 1 & the blow-up at a point of & N. 9 \\
\hline 14 & 5 & 2 & 2 & 1 & $-\infty$ & 1 & the blow-up at a point of & N. 6 \\
\hline 15 & 5 & 3 & 2 & 1 & $-\infty$ & 1 & the blow-up at a point of & N. 7 \\
\hline 16 & 6 & 6 & 1 & 0 & $-\infty$ & 6 & the blow-up at three points of & N. 3 \\
\hline 17 & 6 & 1 & 2 & 1 & $-\infty$ & 2 & the blow-up at two points of & N. 6 \\
\hline 18 & 6 & 2 & 2 & 1 & $-\infty$ & 2 & the blow-up at two points of & N. 7 \\
\hline 19 & 7 & 5 & 1 & 0 & $-\infty$ & 7 & the blow-up at four points of & N. 3 \\
\hline 20 & 7 & 1 & 2 & 1 & $-\infty$ & 3 & the blow-up at three points of & N. 7 \\
\hline 21 & 8 & 4 & 1 & 0 & $-\infty$ & 8 & the blow-up at five points of & N. 3 \\
\hline
\end{tabular}

Table 1. (Continued) 


\begin{tabular}{|c|c|c|c|c|c|c|c|c|}
\hline 22 & 8 & 4 & 3 & 2 & 0 & 0 & $S$ is an abelian surface & - \\
\hline 23 & 8 & 4 & 3 & 1 & 0 & 0 & $S$ is a bielliptic surface & - \\
\hline 24 & 8 & $\leq 3$ & 3 & $\geq 1$ & 1 & 0 & $\begin{array}{c}S \text { is a minimal elliptic surface } \\
\text { with } \chi\left(\mathcal{O}_{S}\right)=0\end{array}$ & - \\
\hline 25 & 8 & 12 & 2 & 0 & $-\infty$ & 4 & $\begin{array}{c}\left(S_{0, e},\left[2 C_{0}+(3+e) f\right]\right), \text { with } \\
e=0,1,2\end{array}$ & - \\
\hline 26 & 8 & 8 & 3 & 1 & $-\infty$ & 0 & $\begin{array}{c}\left(S_{1, e},\left[2 C_{0}+(2+e) f\right]\right), \text { with } \\
e=-1,0,1\end{array}$ & - \\
\hline 27 & 8 & 6 & 3 & 1 & $-\infty$ & 0 & $\left(S_{1,0},\left[3 C_{0}+f\right]\right)$ & - \\
\hline 28 & 8 & 5 & 3 & 1 & $-\infty$ & 0 & $\left(S_{1,-1},\left[5 C_{0}-2 f\right]\right)$ & - \\
\hline 29 & 8 & 4 & 4 & 2 & $-\infty$ & -4 & $\begin{array}{c}\left(S_{2, e},\left[2 C_{0}+(e+1) f\right]\right) \text { with } \\
-2 \leq e \leq 0\end{array}$ & - \\
\hline
\end{tabular}

Table 1. Polarized surfaces $(S, L)$ with $m-d \leq 8$.

[7, Lemma 2.15]); $|L|$ consists of a single divisor, which is the sum of a section and a fiber of the Albanese fibration. As a consequence, [35, Remark 2.3 (2)] implies that no simple blow-up of a pair as in N. 11 can occur. Furthermore, the results concerning ruled surfaces over an elliptic curve, due to Fujita $[6, \S 4]$ (see also [8, Theorem 15.2, cases 0), and 3)-5)]) and Yokoyama [35, Theorem 4.1 (ii)], lead to a simplification in [30, Theorem 4.3]. For instance, combining both we see that for $g=2$ and $S^{\prime}=S_{1, e}$, it must be $v_{i}=1$ for every $i$, hence $e(S)=s=L^{\prime 2}-d$.

REMARK 1. If $L$ is very ample, the only surviving cases in Table 1 are N. $1-5,12,16,19,21,25$, and 26 with $e=-1$.

It is useful to recall that for a polarized surface $(S, L)$ we have $m=$ $e(S)+2 K_{S} L+3 L^{2}$, hence

$$
m-d=e(S)+2 K_{S} L+2 d=e(S)+4(g-1) .
$$

Lemma 1. Let $S$ be a smooth surface, $L$ an ample line bundle on $S$, and let $g:=g(S, L)$ be the sectional genus of $(S, L)$. Suppose that $\sigma: S \rightarrow S_{0}$ is the blow-up of a smooth surface $S_{0}$ at a single point and let $E$ be the exceptional curve. Then there exists an ample line bundle $L_{0}$ on $S_{0}$ such that $L=$ $\sigma^{*} L_{0}-r E$, where $r=L E \geq 1$. Moreover, $L^{2}=L_{0}^{2}-r^{2}, L K_{S}=L_{0} K_{S_{0}}+r$. In particular,

i) $g=g\left(S_{0}, L_{0}\right)-\left(\begin{array}{l}r \\ 2\end{array}\right)$ (hence $g=g\left(S_{0}, L_{0}\right)$ if and only if $r=1$ );

ii) If $L K_{S}=1$, then $S$ cannot have Kodaira dimension $\kappa(S) \geq 1$. 
Proof. The Nakai-Moishezon criterion proves the ampleness of $L_{0}$. Assertion i) is obvious since $K_{S}=\sigma^{*} K_{S_{0}}+E$. To prove assertion ii) note that

$$
1=L K_{S}=L_{0} K_{S_{0}}+r .
$$

We know that $r \geq 1$. If $\kappa(S) \geq 1$, then a suitably high multiple of $K_{S_{0}}$ is effective and nontrivial and then also the first summand on the right hand is positive, due to the ampleness of $L_{0}$, but this gives a contradiction.

Note that ampleness and spannedness of $L$ imply $h^{0}(L) \geq 3$ and $L^{2} \geq 3$ up to well known cases. More precisely, we have also the following

LEMMA 2. Let $L$ be an ample and spanned line bundle on a smooth surface $S$. Then $d=L^{2} \geq 3$ unless $(S, L, e(S), g, m, m-d)$ is one of the following:

i) $\left(\mathbb{P}^{2}, \mathcal{O}_{\mathbb{P}^{2}}(1), 3,0,0,-1\right)$;

ii) $\left(\mathbb{Q}^{2}, \mathcal{O}_{\mathbb{Q}^{2}}(1), 4,0,2,0\right)$;

iii) There exists a morphism $\pi: S \rightarrow \mathbb{P}^{2}$ of degree 2, branched along a smooth curve $\Delta \in\left|\mathcal{O}_{\mathbb{P}^{2}}(2 b)\right|$ for some integer $b \geq 2$ (case $b=1$ fits into case ii)); moreover, $L=\pi^{*} \mathcal{O}_{\mathbb{P}^{2}}(1), e(S)=2\left(2 b^{2}-3 b+3\right), g=b-1$, $m=2 b(2 b-1) \geq 12$ and $m-d=2\left(2 b^{2}-b-1\right) \geq 10$.

Proof. It is enough to consider the morphism defined by $|L|$ and recall that $L^{2}$ is the product of its degree and the degree of the image. In case iii) note that $\pi^{*}\left|\mathcal{O}_{\mathbb{P}^{2}}(1)\right|=|L|$ (since $b \geq 2$ ). Recall that $\pi_{*} \mathcal{O}_{S}=\mathcal{O}_{\mathbb{P}^{2}} \oplus \mathcal{O}_{\mathbb{P}^{2}}(-b)$. Since $\Delta \in\left|\mathcal{O}_{\mathbb{P}^{2}}(2 b)\right|$ and $K_{S}=\pi^{*}\left(K_{\mathbb{P}^{2}}+\frac{1}{2} \Delta\right)=\pi^{*}\left(\mathcal{O}_{\mathbb{P}^{2}}(b-3)\right)$, projection formula gives

$$
h^{0}\left(K_{S}\right)=h^{0}\left(\pi_{*} K_{S}\right)=h^{0}\left(\mathcal{O}_{\mathbb{P}^{2}}(b-3) \oplus \mathcal{O}_{\mathbb{P}^{2}}(-3)\right)=h^{0}\left(\mathcal{O}_{\mathbb{P}^{2}}(b-3)\right)=\left(\begin{array}{c}
b-1 \\
2
\end{array}\right) .
$$

Similarly, $h^{1}\left(K_{S}\right)=0$ and then, since $K_{S}^{2}=2(b-3)^{2}$, Noether's formula allows us to compute $e(S)$. The value of $g$ is provided by the Riemann-Hurwitz formula, by restricting $\pi$ to a general element of $\pi^{*}\left|\mathcal{O}_{\mathbb{P}^{2}}(1)\right|$.

The following fact will be used often.

REMARK 2. Let $(S, L)$ be a smooth polarized surface of sectional genus $g \geq 2$. If $S$ is ruled, but $(S, L)$ is not a scroll, then $g \geq 2 q$. Actually, due to the assumptions, $K_{S}+L$ is nef, hence $\left(K_{S}+L\right)^{2} \geq 0$. Moreover, $K_{S}^{2} \leq$ $8(1-q)$. Combining these inequalities we get

$$
\begin{aligned}
0<L^{2} & \leq L^{2}+\left(K_{S}+L\right)^{2}=2\left(K_{S}+L\right) L+K_{S}^{2} \\
& \leq 4(g-1)+8(1-q)=4(g-2 q+1) .
\end{aligned}
$$

Therefore $g>2 q-1$. 
Now, observe that for a polarized surface $(S, L)$, the inequality $m-d \geq 2 g$ in [30, Proposition 3.2] can be further explored, as the following result shows.

THEOREM 1. Let $(S, L)$ be a smooth polarized surface and put $m:=$ $c_{2}\left(J_{1}(L)\right)$. Then

(A) $m-d=2 g+1$ if and only if either

( $\alpha) \quad(m-d, g)=(3,1),(5,2)$ and $(S, L)$ is as in Table 1 , or

(B) $g=2 q \geq 4, S$ is the blowing-up $\sigma: S \rightarrow S_{q, e}$ of $S_{q, e}$ at a point $p$,

$L=\sigma^{*} L^{\prime}-\sigma^{-1}(p)$ and $L^{\prime} \equiv\left[2 C_{0}+(e+1) f\right]$.

(B) $m-d=2 g+2$ if and only if either

$(\gamma) \quad(m-d, g)=(4,1),(6,2),(8,3)$ and $(S, L)$ is as in Table 1 , or

( $\delta) \quad g \geq 4$ and $(S, L)$ is one of the following polarized surfaces:

$\left(\delta_{1}\right) \quad S=S_{q, e}$ with $q \geq 2, g=2 q+1, L \equiv\left[2 C_{0}+(e+2) f\right]$ and $d=8$;

$\left(\delta_{2}\right) \quad S=S_{2,-1}, g=5, L \equiv\left[3 C_{0}-f\right]$ and $d=3$;

$\left(\delta_{3}\right) \quad S$ is the blowing-up $\sigma: S \rightarrow S_{q, e}$ of $S_{q, e}, q \geq 2, e \leq 0$, at two points $p_{1}, p_{2}$, lying on distinct fibers, $g=2 q$, $L=\sigma^{*} L^{\prime}-\sigma^{-1}\left(p_{1}\right)-\sigma^{-1}\left(p_{2}\right), L^{\prime} \equiv\left[2 C_{0}+(e+1) f\right]$ and $d=2$.

For the proof we need the following lemma, consequence of a nefness result for the relative adjoint bundle on polarized fibrations [10, Sec. 1].

LEMMA 3. Let $(S, L)$ be a polarized ruled surface which is not a scroll and suppose that $q \geq 2$. Let $\pi: S \rightarrow B$ be the ruling projection and set $a:=L F>1$, where $F$ is a fiber of $\pi$. If either $g=2 q$ or $g=2 q+1$, then $a \leq 3$, equality implying $q=2$.

Proof. Clearly $a \geq 2$. Let $K_{S / B}$ be the relative canonical bundle. Since $(S, L)$ is not a scroll,

the line bundle $K_{S / B}+L$ is nef,

according to [10, Theorem 1.1.2]. Note that

$$
\left(K_{S / B}+L\right) L=\left(K_{S}+L\right) L-\pi^{*} K_{B} L=2 g-2-(2 q-2) a .
$$

Then, due to (1) and (2), letting $g=2 q$ we have $0 \leq\left(K_{S / B}+L\right) L=2(q+$ $(q-1)(1-a))$, hence $a \leq 1+q /(q-1) \leq 3$ since $q \geq 2$, and $a=3$ implies $q=2$. Next, suppose that $g=2 q+1$ and $a \geq 3$. Observe that if $\left(K_{S / B}+L\right) L$ $=0$, then $K_{S / B}+L \equiv 0$ due to the Hodge index theorem combined with (1), but this is impossible, because $\left(K_{S / B}+L\right) F=-2+a \geq 1$. Therefore, in view of (1) and (2), letting $g=2 q+1$ in (2), we have $0<\left(K_{S / B}+L\right) L=2(2 q+1)-$ $2-(2 q-2) a=2(2 q-a(q-1))$. Thus $\quad a<2 q /(q-1)=2+2 /(q-1) \leq 4$ since $q \geq 2$, and then $a \leq 3$, equality implying $q=2$. 
Proof (of Theorem 1). Case (A). We can assume $g \geq 4$, since otherwise $m-d \leq 7$, hence $(S, L)$ is as in Table 1 , which leads to $(\alpha)$. Then (\#) implies $0=e(S)+2 g-5 \geq e(S)+3$, hence $S$ is a ruled surface. Note that $(S, L)$ is not a scroll since $m-d \neq 0$, hence $g \geq 2 q$ by Remark 2. Let $\sigma: S \rightarrow S^{\prime}$ be the blowing-up of a smooth ruled surface $S^{\prime}$ at a finite set of points $\mathfrak{B} \subset S^{\prime}$. Denote by $s$ the cardinality of $\mathfrak{B}$. Thus $e(S)=4(1-q)+s$ and this gives $0=$ $2(g-2 q)+(s-1)$. Observe that necessarily $s \leq 1$, and $s=0$ cannot occur. Hence $s=1$, i.e. $S$ is the blowing-up of $S^{\prime}$ at a single point, $S^{\prime}$ is a geometrically ruled surface $S_{q, e}$ over a smooth curve of genus $q$ for some $e$, and $g=2 q$. As a consequence, $q \geq 2$. Letting $a=L F$, where $F$ is a fiber of $S$, we can thus apply Lemma 3 , obtaining that either (i) $a=2$, or (ii) $a=3$ and $q=2$. Moreover, write $L=\sigma^{*} L^{\prime}-v E$, where $E$ is the exceptional divisor contracted by $\sigma, L^{\prime}$ is an ample divisor on $S^{\prime}$ and $v$ is a positive integer. Since $S^{\prime}=S_{q, e}$ and $F$ is the proper transform of a fiber $f$ of $S^{\prime}$ via $\sigma$, we can write $L^{\prime} \equiv\left[a C_{0}+b f\right]$. Note that

$$
1 \leq\left(\sigma^{*} f-E\right)\left(\sigma^{*} L^{\prime}-v E\right)=\left(\sigma^{*} f-E\right)\left(\sigma^{*}\left(a C_{0}+b f\right)-v E\right)=a-v,
$$

i.e. $1 \leq v \leq a-1$. Thus in case (i) we have $v=1$ and since $S^{\prime}=S_{q, e}$, by the genus formula we deduce that

$$
4 q-2=2 g-2=\left(K_{S}+L\right) L=\left(K_{S^{\prime}}+L^{\prime}\right) L^{\prime}=4(q-1)+(2 b-2 e),
$$

i.e. $b=e+1$. This gives case $(\beta)$ in the statement.

Finally, in case (ii) from $q=2$ we see that $g=4$ and $1 \leq v \leq 2$. By the genus formula we thus get the following relation

$$
v(v-1)=2(2 b-3 e) .
$$

According to it, for $v=1$ we get $b=3 e / 2$. Therefore the ampleness of $L^{\prime}$ $[15$, p. 382] leads to a contradiction regardless of the value of $e$. On the other hand, for $v=2$ we get $b=(3 e+1) / 2$ and then the ampleness of $L^{\prime}$ combined with the Nagata inequality $e \geq-q=-2$ and the fact that $b$ must be an integer, implies $e=-1$, hence $S^{\prime}=S_{2,-1}$ and $L^{\prime} \equiv\left[3 C_{0}-f\right]$; in this case, however, $L^{2}=L^{\prime 2}-4=9-6-4<0$, contradicting the ampleness of $L$. Hence case (ii) cannot occur.

Case (B). If $g \leq 3$, Table 1 leads to $(\gamma)$. So, as in case (A), we can suppose that $g \geq 4$. Then $0=e(S)+2 g-6 \geq e(S)+2$, hence $S$ is a ruled surface. Moreover, note that $(S, L)$ is not a scroll over a curve. Hence $g \geq 2 q$ by Remark 2 again and using the same notation as in case (A), we can write $e(S)=4(1-q)+s$ for some integer $s \geq 0$. This gives $0=2(g-2 q)+$ $(s-2)$, i.e. $0 \leq s \leq 2$. Thus we have either $(\mathrm{j}) s=0, g=2 q+1$, or $(\mathrm{jj}) s=2$, $g=2 q$. In both cases, $q \geq 2$. 
In case $(\mathrm{j}), S=S_{q, e}$ is a geometrically ruled surface over a smooth curve of genus $q$. Note that $4 \leq g=2 q+1$ implies $q \geq 2$. We can write $L \equiv$ $\left[a C_{0}+b f\right]$ with $a \geq 2$, since $(S, L)$ is not a scroll. Then

$$
L^{2}=a(2 b-a e) \text {. }
$$

By Lemma 3 we know that either $\left(\mathrm{j}_{1}\right) a=2$, or $\left(\mathrm{j}_{2}\right) a=3$ and $q=2$. In case $\left(\mathrm{j}_{1}\right)(S, L)$ is a conic bundle, hence $\left(K_{S}+L\right)^{2}=0$. Thus

$$
d=L^{2}=2\left(K_{S}+L\right) L+K_{S}^{2}=4(g-1)+8(1-q)=4(g-2 q)+4=8,
$$

which gives $L \equiv\left[2 C_{0}+(e+2) f\right]$; moreover, $-q \leq e \leq 1$ in view of the Nagata inequality and the ampleness conditions $\left[15\right.$, p. 382]. This gives case $\left(\delta_{1}\right)$ of the statement.

In case $\left(\mathrm{j}_{2}\right)$, since $q=2$ and $g=2 q+1$, recalling the expression of $K_{S}$ and the ampleness of $L$, we obtain $\left(K_{S}+L\right)^{2}=2 b-3 e+4 \geq 5$. Thus

$$
\begin{aligned}
L^{2} & \leq L^{2}+\left(K_{S}+L\right)^{2}-5=2\left(K_{S}+L\right) L+K_{S}^{2}-5 \\
& =4(g-1)+8(1-q)-5=3 .
\end{aligned}
$$

On the other hand, the expression of $L^{2}$ combined with the ampleness of $L$ shows that $L^{2} \geq 3$. Therefore $d=L^{2}=3$ and $b=\frac{1}{2}(3 e+1)$. This implies that $e$ is odd; moreover the ampleness conditions for $L$ show that it is negative. Recalling Nagata inequality we thus get $e=-1$, hence $b=-1$. This gives case $\left(\delta_{2}\right)$ in the statement.

In case (jj), $S$ is obtained by a blowing-up $\sigma: S \rightarrow S^{\prime}$ of a geometrically ruled surface $S^{\prime}=S_{q, e}$ at two points $p_{1}$ and $p_{2}$. Denote by $E_{i}$ the corresponding exceptional divisor for $i=1,2$. Thus $L=\sigma^{*} L^{\prime}-v_{1} E_{1}-v_{2} E_{2}$ for an ample line bundle $L^{\prime}=\left[a C_{0}+b f\right]$ on $S^{\prime}$ with $a \geq 2$ and positive integers $v_{i}$ for $i=1,2$. If $a=2$, the ampleness of $L$ implies that $p_{1}$ and $p_{2}$ lie on distinct fibers and $v_{i}=1, i=1,2$. From

$$
4 q-2=2 g-2=\left(K_{S}+L\right) L=\left(K_{S^{\prime}}+L^{\prime}\right) L^{\prime}=4(q-1)+2(b-e)
$$

we get $b=e+1$, hence $e \leq 0$ in view of the ampleness conditions. Then $d=L^{2}=L^{\prime 2}-2=2$. This gives case $\left(\delta_{3}\right)$ in the statement. If $a>2$, then necessarily $a=3$ and $q=2$ by Lemma 3 . If $f_{0}$ is the fiber containing $p_{i}$, the ampleness of $L$ implies $1 \leq\left(\sigma^{*} f_{0}-E_{i}\right) L=3-v_{i}$, hence $v_{i} \leq 2$, that is, $v_{i}=$ 1,2 . Since $q=2$, we have $g=4$ and by the genus formula we obtain that

$$
\begin{aligned}
6=2 g-2=\left(K_{S}+L\right) L= & (a-2)(b-e a)+a(b+2-e)-v_{1}\left(v_{1}-1\right) \\
& -v_{2}\left(v_{2}-1\right) \\
= & b-3 e+3(b+2-e)-v_{1}\left(v_{1}-1\right)-v_{2}\left(v_{2}-1\right),
\end{aligned}
$$


i.e.

$$
(2 b-3 e)=\frac{1}{2}\left(v_{1}\left(v_{1}-1\right)+v_{2}\left(v_{2}-1\right)\right) .
$$

Note that $0<d=3(2 b-3 e)-v_{1}^{2}-v_{2}^{2}$, but this leads to a numerical contradiction.

If $S$ is not a ruled surface, a result of Serrano [32] allows us to go further.

Proposition 1. Let $(S, L)$ be a smooth polarized surface and put $m:=$ $c_{2}\left(J_{1}(L)\right)$. Suppose that $m-d>0$ and assume that $S$ is not a ruled surface. Then $m-d \geq 2 g+d$ unless one of the following cases occurs:

(1) $S$ is an abelian or a bielliptic surface and $m-d=2 g+d-2$;

(2) $S$ is an elliptic quasi-bundle $f: S \rightarrow B$ over a smooth curve $B$ of genus $g(B) \leq 1, q=1, p_{g}(S)=0$ and $m-d=2 g+d-1$; moreover, $f$ has only multiple fibers $m_{i} F_{i}, i=1, \ldots, s$, as singular fibers, where $F_{i}$ is a smooth elliptic curve, and letting $F$ denote the general fiber of $f$, one of the following holds:

(a) $g(B)=1, s=1, m_{1}=2$ and $F L=2$ (e.g., see [9]);

(b) $g(B)=0$ and $\left(m_{1}, \ldots, m_{s}\right)=(2,2,2,2,2),(4,4,4),(2,6,6)$ with $F L=2,4,6$ respectively (e.g., see [32]).

Proof. Assume that $m-2 d \leq 2 g-1$. Then

$$
e(S)+2 g-2+K_{S} L=m-2 d \leq 2 g-1,
$$

i.e. $e(S)+K_{S} L \leq 1$. Note that $e(S) \geq 0$ and $K_{S} L \geq 0$ since $S$ is not a ruled surface. Thus we get the following three cases:

(i ) $e(S)=K_{S} L=0$ and $m-2 d=2 g-2$;

(ii) $e(S)=0, K_{S} L=1$ and $m-2 d=2 g-1$;

(iii) $e(S)=1, K_{S} L=0$ and $m-2 d=2 g-1$.

Case (iii) cannot occur: actually, it follows from $K_{S} L=0$ that $K_{S}$ is numerically trivial, since $S$ is not a ruled surface. Therefore $S$ is a minimal surface with $\kappa(S)=0$, but this contradicts $e(S)=1$. In cases (i) and (ii), $S$ is a minimal surface with $\kappa(S) \leq 1$, since $e(S)=0$. If $\kappa(S)=1$ then a multiple of the canonical bundle is nontrivial and effective, but this contradicts (i) by the ampleness of $L$. Moreover, in case (ii), since $K_{S}$ is not numerically trivial, we see that $S$ is a properly elliptic minimal surface over a smooth curve $B$, hence $K_{S}^{2}=0$. Thus $\chi\left(\mathcal{O}_{S}\right)=0$, by the Noether's formula. Then, by [33, Proposition 4.2], the elliptic fibration $f: S \rightarrow B$ is a quasi-bundle, i.e. any singular fiber is a multiple of a smooth elliptic curve [33, Definition 1.1]. By combining the canonical bundle formula for an elliptic fibration with the condition $L K_{S}=1$, it thus follows that $f$ necessarily has some multiple fiber and 
$g(B) \leq 1$. Moreover, $0<p_{g}(S)+1=q=g(B)$ or $g(B)+1[33, \S 4]$, but the latter case cannot occur if $g(B)=1$, due to the Katsura-Ueno property [33, Proposition 4.3]. Then the assertion follows from [32], taking into account that this result only depends on the condition $L K_{S}=1$ (and not $g=2$ ), as pointed out in [33, final comment at p. 300].

REMARK 3. Let $S$ be a surface of general type. Then, by combining Noether's formula with the Bogomolov-Miyaoka-Yau inequality, we have $e(S) \geq 3$.

\section{Triplets $(X, \mathscr{E}, H)$ with low $m_{2}$}

Our basic setting from here on is the following:

$X$ is a smooth complex projective variety of dimension $n, \mathscr{E}$ is an ample vector bundle of rank $r$ on $X$ with $2 \leq r \leq n-2$ and $H$ is an ample line bundle on $X$. Furthermore, the ample vector bundle of rank $n-2$ on $X$ given by $\mathscr{F}:=$ $\mathscr{E} \oplus H^{\oplus(n-r-2)}$ has a section vanishing on a smooth surface $S \subset X$.

REMARK 4. A concrete way to fit into $(\diamond)$ for $r<n-2$ is to consider the following slightly more special setting: $X$ is a smooth complex projective variety of dimension $n, \mathscr{E}$ is an ample vector bundle of rank $r$ on $X$ with $2 \leq$ $r<n-2$, having a section whose zero locus is a smooth subvariety $Z \subset X$ of the expected dimension $n-r$ (which happens, e.g., if $\mathscr{E}$ is spanned), and $H$ is an ample line bundle on $X$ such that $\operatorname{Tr}_{Z}|H|$ (the trace of $|H|$ on $Z$ ) is base point free.

Note that in this setting the line bundle $H_{Z}$ is spanned "a fortiori". Clearly this fits into $(\diamond)$ simply letting $S$ denote the surface cut out by $n-r-2$ general elements of $\operatorname{Tr}_{Z}|H|$. Actually, if $\sigma \in \Gamma(X, \mathscr{E})$ defines $Z$, there are sections $s_{i} \in \Gamma(X, H)$ whose restrictions to $Z$ define a smooth surface

$$
S:=\bigcap_{i=1}^{n-r-2}\left(\left.s_{i}\right|_{Z}\right)_{0},
$$

which is the zero locus of the section $\left(\sigma, s_{1}, \ldots, s_{n-r-2}\right) \in \Gamma(X, \mathscr{F})$.

In Subsection 4.1 we will add to $(\diamond)$ the requirement that

$$
H_{S} \text { is spanned. }
$$

Clearly, this condition is trivially satisfied in the setting of Remark 4 since, as noted, $H_{Z}$ is spanned. Furthermore, in Subsection 4.2 we will put the stronger 
requirement that

$$
H_{S} \text { is very ample. }
$$

Assuming that $(X, \mathscr{E}, H)$ is as in $(\diamond)$, we set

$$
d:=H_{S}^{2}=H^{2} \cdot c_{n-2}=c_{r}(\mathscr{E}) \cdot H^{n-r} \quad \text { and } \quad g:=g\left(S, H_{S}\right) .
$$

This notation is consistent with that used in Section 2 since $d=H_{S}^{2}$ and $g$ are the degree and the sectional genus of the polarized surface $(S, L):=\left(S, H_{S}\right)$, respectively. Moreover, we have the following technical result.

Proposition 2. Let $(X, \mathscr{E}, H)$ and $S$ be as in $(\diamond)$ (see Introduction). If $m_{2}=m_{2}(X, \mathscr{E}, H)$ is as in $(*)$, then

$$
m_{2}=c_{2}\left(J_{1}\left(H_{S}\right)\right)
$$

Proof. Consider the dual of the tangent-normal bundle sequence of $S \subset X$

$$
0 \rightarrow \mathscr{N}_{S / X}^{\vee} \cong \mathscr{F}_{S}^{\vee} \rightarrow\left(\Omega_{X}\right)_{S} \rightarrow \Omega_{S} \rightarrow 0 .
$$

It fits into the following diagram:

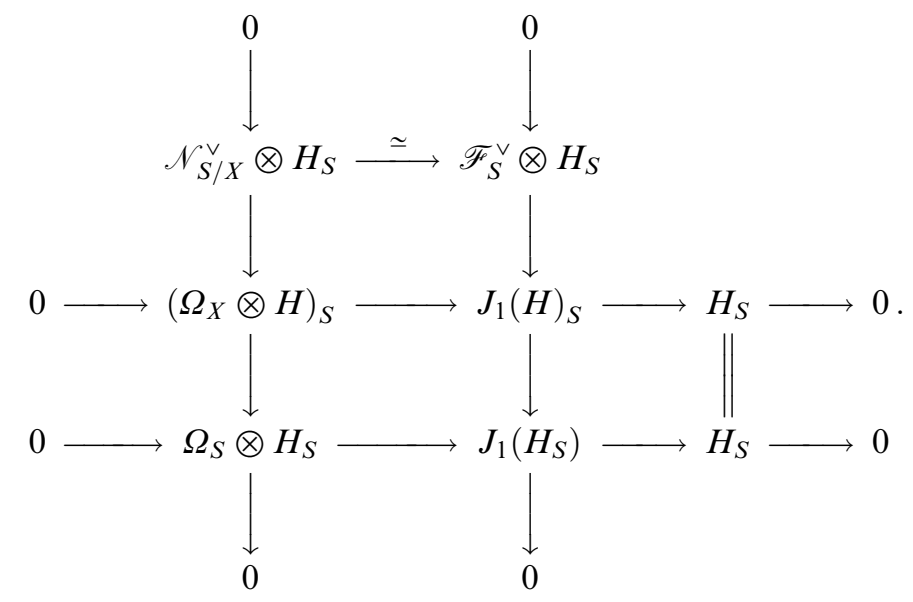

Then, recalling $(*)$ we get

$$
\begin{aligned}
m_{2}=m_{2}(X, \mathscr{E}, H)= & {\left[c_{2}\left(\Omega_{X} \oplus c_{1}\right)+c_{1}^{2}-c_{2}+H^{2}\right]_{S}+4(g-1) } \\
= & {\left[c_{2}\left(\Omega_{X S}\right)+c_{1}\left(\Omega_{X S}\right) c_{1}\left(\mathscr{F}_{S}\right)+c_{1}\left(\mathscr{F}_{S}\right)^{2}-c_{2}\left(\mathscr{F}_{S}\right)\right] } \\
& +H_{S}^{2}+4\left(g\left(S, H_{S}\right)-1\right)
\end{aligned}
$$




$$
\begin{aligned}
= & {\left[c_{2}\left(\Omega_{X S}\right)-c_{1}\left(\Omega_{S}\right) c_{1}\left(\mathscr{F}_{S}^{\vee}\right)-c_{2}\left(\mathscr{F}_{S}^{\vee}\right)\right]+H_{S}^{2} } \\
& +4\left(g\left(S, H_{S}\right)-1\right) \\
= & c_{2}\left(\Omega_{S}\right)+H_{S}^{2}+2\left(K_{S}+H_{S}\right) H_{S} \\
= & {\left[c_{2}\left(\Omega_{S}\right)+c_{1}\left(\Omega_{S}\right) H_{S}+H_{S}^{2}\right]+\left[\left(K_{S}+2 H_{S}\right) H_{S}\right] } \\
= & c_{2}\left(\Omega_{S} \otimes H_{S}\right)+c_{1}\left(\Omega_{S} \otimes H_{S}\right) H_{S} \\
= & c_{2}\left(J_{1}\left(H_{S}\right)\right) .
\end{aligned}
$$

Lemma 4. Suppose that there exists an effective divisor $E \cong \mathbb{P}^{n-1}$ on $X$ such that

$$
\left(E, \mathscr{F}_{E}, E_{E}\right) \cong\left(\mathbb{P}^{n-1}, \mathcal{O}_{\mathbb{P}^{n-1}}(1)^{\oplus(n-2)}, \mathcal{O}_{\mathbb{P}^{n-1}}(-1)\right) .
$$

If $q>0$, then $S$ is not ruled.

Proof. Suppose that $\kappa(S)=-\infty$ and note that $S \neq \mathbb{P}^{2}$. Let $f: X \rightarrow X^{\prime}$ be the contraction of $E$. Then by [20, Lemma 5.1] and [17, Lemma 2.2] we know that there exist an ample vector bundle $\mathscr{F}^{\prime}$ of rank $n-2$ on $X^{\prime}$ and a section $s^{\prime} \in \Gamma\left(\mathscr{F}^{\prime}\right)$ such that $\mathscr{F} \cong f^{*} \mathscr{F}^{\prime} \otimes \mathcal{O}_{X}(-E), S^{\prime}:=\left(s^{\prime}\right)_{0}$ is a smooth surface and $\left.f\right|_{S}: S \rightarrow S^{\prime}$ is a birational morphism which contracts the (-1)curve $\left.E\right|_{S}$. Since $K_{S^{\prime}}=\left[K_{X^{\prime}}+\operatorname{det} \mathscr{F}^{\prime}\right]_{S^{\prime}}$ is not nef, $S^{\prime}$ being ruled, and $q\left(S^{\prime}\right)>0$, [28, Theorem] implies one of the following possibilities:

(i) there exists an effective divisor $E^{\prime}$ on $X^{\prime}$ such that

$$
\left(E^{\prime}, \mathscr{F}_{E^{\prime}}^{\prime}, E_{E^{\prime}}^{\prime}\right) \cong\left(\mathbb{P}^{n-1}, \mathcal{O}_{\mathbb{P}^{n-1}}(1)^{\oplus(n-2)}, \mathcal{O}_{\mathbb{P}^{n-1}}(-1)\right) ;
$$

(ii) there is a surjective morphism $\varphi: X^{\prime} \rightarrow W$ expressing $X^{\prime}$ either (a) as a $\mathbb{P}^{t}$-bundle over a smooth variety $W$ of dimension $\operatorname{dim} W \leq 2$, or (b) as a quadric fibration over a smooth curve $W$.

We show that case (ii) does not occur. If (ii) holds, then $X^{\prime}$ is covered by lines. Note that any line of $X^{\prime}$ is contained in a fiber of $\varphi$ since $q\left(X^{\prime}\right)=$ $q\left(S^{\prime}\right)=q>0$. Suppose that $p^{\prime}=f(E)$ lies on a smooth fiber $F^{\prime}$ of $\varphi$, so that $F^{\prime}$ is either $\mathbb{P}^{t}$ with $t=n-1, n-2$, or $\mathbb{Q}^{n-1}$; take a line $l^{\prime}$ passing through $p^{\prime}$ and consider its proper transform $l$ via $f$. Then

$$
\mathscr{F}_{l} \cong\left(f^{*} \mathscr{F}^{\prime} \otimes \mathcal{O}_{X}(-E)\right)_{l} \cong f^{*}\left(\mathscr{F}_{l^{\prime}}^{\prime}\right) \otimes \mathcal{O}_{l}(-1) \cong f^{*}\left(\mathscr{F}_{F^{\prime}}^{\prime}\right)_{l^{\prime}} \otimes \mathcal{O}_{l}(-1) .
$$

From [28, Theorem, cases $(10)-(13)]$ we see that $(n-3)$ summands at least of $\left(\mathscr{F}_{F^{\prime}}^{\prime}\right)$ are $\mathcal{O}_{F^{\prime}}(1)$. Since $n \geq 4$, it thus follows that $\mathscr{F}_{l}$ has a summand which is $\mathcal{O}_{l}$, but this contradicts the ampleness of $\mathscr{F}$. On the other hand, if $F^{\prime}$ is a singular fiber of $\varphi$, i.e. a quadric cone with vertex a point, then we have $\operatorname{deg} \mathscr{F}_{l^{\prime}}^{\prime}=\operatorname{deg} \mathscr{F}_{\lambda^{\prime}}^{\prime}=n-2$ for some line $\lambda^{\prime}$ contained in a smooth fiber [28, 
Theorem, case (12)]. By the ampleness of $\mathscr{F}^{\prime}$ we get $\mathscr{F}_{l^{\prime}}^{\prime}=\mathcal{O}_{l^{\prime}}(1)^{\oplus(n-2)}$. Thus the same argument as above applies and we conclude that this case cannot occur as well. Finally, if we are in case (i), by a recursive argument we get a contradiction.

Lemma 5. Let $(X, \mathscr{E}, H)$ be as in $(\diamond)$, let $\mathscr{F}=\mathscr{E} \oplus H^{\oplus(n-r-2)}$, and suppose that $S$ is a $\mathbb{P}^{1}$-bundle over a smooth curve $B$ of positive genus. Then $X$ is a $\mathbb{P}^{n-1}$-bundle over $B$, with the projection $p: X \rightarrow B$ inducing the ruling of $S$, and $\mathscr{F}_{F}=\mathcal{O}_{\mathbb{P}^{n-1}}(1)^{\oplus(n-2)}$ for every fiber $F \cong \mathbb{P}^{n-1}$ of $p$. In particular, either $r<$ $n-2$ and $\left(S, H_{S}\right)$ is a scroll, or $r=n-2$ and $H_{F}=\mathcal{O}_{\mathbb{P}^{n-1}}(t)$ with $t=H_{S} f$, $f$ being any fiber of $S$. Conversely, if $(X, \widetilde{F})$ satisfies the above conditions, then $S$ is a $\mathbb{P}^{1}$-bundle over $B$; moreover, $\left(S, H_{S}\right)$ is a scroll if either $r<n-2$ or $H_{F}=\mathcal{O}_{\mathbb{P}^{n-1}}(1)$.

Proof. The description of $(X, \mathscr{F})$, including the fibration $p: X \rightarrow B$, follows from [20, Theorem]. If $r<n-2$, then $H_{F}=\mathcal{O}_{\mathbb{P}^{n-1}}(1)$, being a summand of $\mathscr{F}_{F}$, and then $\left(S, H_{S}\right)$ is a scroll. On the other hand, if $r=n-2$ then $\mathscr{F}=\mathscr{E}$, so we have no information on $H$. We can write $H_{F}=\mathcal{O}_{\mathbb{P}^{n-1}}(t)$ for some positive integer $t$. Since the ruling of $S$ is induced by $p: X \rightarrow B$ any fiber $f$ of $S$ is a line, being the zero locus of a section of $\mathscr{E}_{F}=\mathcal{O}_{\mathbb{P}^{n-1}}(1)^{\oplus(n-2)}$, where $F=\mathbb{P}^{n-1}$ is the corresponding fiber of $X$. Thus the assertion follows from the equality

$$
H_{S} f=H_{F} \cdot\left(\mathcal{O}_{\mathbb{P}^{n-1}}(1)\right)^{n-2}=t .
$$

The converse is obvious.

Recall the notation $\delta:=m_{2}-d$. As a first thing, let us characterize the inequality $\delta<0$.

THeOREM 2. Let $(X, \mathscr{E}, H)$ be as in $(\diamond)$. Then $\delta \geq 0$ unless $(X, \mathscr{E}, H)$ is either $\left(\mathbb{P}^{n}, \mathcal{O}_{\mathbb{P}^{n}}(1)^{\oplus r}, \mathcal{O}_{\mathbb{P}^{n}}(1)\right)\left(m_{2}=0\right)$, or $\left(\mathbb{P}^{n}, \mathcal{O}_{\mathbb{P}^{n}}(1)^{\oplus(n-2)}, \mathcal{O}_{\mathbb{P}^{n}}(2)\right)\left(m_{2}=3\right)$. Moreover, equality holds if and only if $(X, \mathscr{E}, H)$ is one of the following:

(1) $\left(\mathbb{P}^{n}, \mathcal{O}_{\mathbb{P}^{n}}(2) \oplus \mathcal{O}_{\mathbb{P}^{n}}(1)^{\oplus(r-1)}, \mathcal{O}_{\mathbb{P}^{n}}(1)\right) ; \quad\left(m_{2}=2\right)$

(2) $\left(\mathbb{Q}^{n}, \mathcal{O}_{\mathbb{Q}^{n}}(1)^{\oplus r}, \mathcal{O}_{\mathbb{Q}^{n}}(1)\right) ; \quad\left(m_{2}=2\right)$

(3) $X$ is a $\mathbb{P}^{n-1}$-bundle over a smooth curve $B, \mathscr{E}_{F}=\mathcal{O}_{\mathbb{P}^{n-1}}(1)^{\oplus r}$ and $H_{F}=$ $\mathcal{O}_{\mathbb{P}^{n-1}}(1)$, for every fiber $F=\mathbb{P}^{n-1}$ of the bundle projection $\pi: X \rightarrow B$ and $\left(S, H_{S}\right)$ is a scroll over $B$ via $\left.\pi\right|_{S}: S \rightarrow B . \quad\left(m_{2}=d:=H_{S}^{2}\right)$

Proof. Since $(\diamond)$ holds, by Proposition 2 and [25, Proposition (A.1)] we see that $\delta=c_{2}\left(J_{1}\left(\left.H\right|_{S}\right)\right)-d \geq 0$ except for (a) $\left(S, H_{S}\right)=\left(\mathbb{P}^{2}, \mathcal{O}(e)\right)$ with $e=1,2$, and $\delta=0$ holds if and only if (b) $\left(S, H_{S}\right)$ is a scroll over a smooth curve. 
In (a), by [19, Theorem A] we know that $X=\mathbb{P}^{n}$ and $\mathscr{F}=\mathcal{O}(1)^{\oplus(n-2)}$, which gives rise to the first two triplets in the statement.

In (b), by [21, Theorem 2] we see that $(X, \mathscr{F})$ is one of the following pairs:

(i ) $\left(\mathbb{P}^{n}, \mathcal{O}(1)^{\oplus(n-3)} \oplus \mathcal{O}(2)\right)$;

(ii) $\left(\mathbb{Q}^{n}, \mathcal{O}(1)^{\oplus(n-2)}\right)$;

(iii) $\quad X$ is a $\mathbb{P}^{n-1}$-bundle over a smooth curve $B$ and $\mathscr{F}_{F}=\mathcal{O}_{\mathbb{P}^{n-1}}(1)^{\oplus(n-2)}$ for every fiber $F=\mathbb{P}^{n-1}$ of the bundle projection.

Since in this situation $S \neq \mathbb{P}^{2}$, cases (i) and (ii) give (1) and (2) of the statement with $\mathscr{E}=\mathcal{O}(1)^{\oplus(r-1)} \oplus \mathcal{O}(2)$ and $\mathscr{E}=\mathcal{O}(1)^{\oplus r}$ respectively and $H=\mathcal{O}(1)$ in both cases. Finally, (iii) leads easily to case (3) of the statement.

The following result characterizes the low positive values of $\delta$.

THEOREM 3. Let $(X, \mathscr{E}, H)$ be as in $(\diamond)$, and suppose that $\delta$ is positive. Then

$$
\delta \geq 3
$$

with equality if and only if $(X, \mathscr{E}, H)=\left(\mathbb{P}^{n}, \mathcal{O}_{\mathbb{P}^{n}}(1)^{\oplus(n-2)}, \mathcal{O}_{\mathbb{P}^{n}}(3)\right)\left(m_{2}=12\right)$.

Moreover, if $\delta=4$ then $(X, \mathscr{E}, H)$ is one of the following triplets:

(1) $\quad\left(\mathbb{P}^{n}, \mathcal{O}_{\mathbb{P}^{n}}(2) \oplus \mathcal{O}_{\mathbb{P}^{n}}(1)^{\oplus(n-3)}, \mathcal{O}_{\mathbb{P}^{n}}(2)\right) ; \quad\left(m_{2}=12\right)$

(2) $\left(\mathbb{Q}^{n}, \mathcal{O}_{\mathbb{Q}^{n}}(1)^{\oplus(n-2)}, \mathcal{O}_{\mathbb{Q}^{n}}(2)\right) ; \quad\left(m_{2}=12\right)$

(3) $r=n-2, X$ is a $\mathbb{P}^{n-1}$-bundle over a smooth curve $B$ of genus 1 , $\mathscr{E}_{F}=\mathcal{O}_{\mathbb{P}^{n-1}}(1)^{\oplus(n-2)}$ and $H_{F}=\mathcal{O}_{\mathbb{P}^{n-1}}(t)$, with $t=2$ or 3 , for every fiber $F=\mathbb{P}^{n-1}$ of the bundle projection $X \rightarrow B$; moreover, $\left(S, H_{S}\right)$ is, up to numerical equivalence, either $\left(S_{1,-1},\left[3 C_{0}-f\right]\right)\left(m_{2}=7\right)$ or $\left(S_{1, e}\right.$, $\left.\left[2 C_{0}+(e+1) f\right]\right)$ with $e \in\{-1,0\} \quad\left(m_{2}=8\right)$.

Finally, if $\delta=5$ then $(X, \mathscr{E}, H)$ is one of the following triplets:

(4) there is a vector bundle $\mathscr{T}$ on a smooth curve $C$ of genus one such that $X \cong \mathbb{P}_{C}(\mathscr{T}), H_{F}=\mathcal{O}_{F}(1)$ and $\mathscr{E}_{F} \cong \mathcal{O}_{F}(2) \oplus \mathcal{O}_{F}(1)^{\oplus(r-1)}$ for any fiber $F \cong \mathbb{P}^{n-1}$ of $X \rightarrow C$; moreover, $S$ is the blowing-up $\sigma: S \rightarrow S_{1, e}$ of $S_{1, e} \rightarrow C$ with $e \in\{-1,0\}$ at a point $p$ and $H_{S} \equiv\left[\sigma^{*}\left(2 C_{0}+\right.\right.$ $\left.(e+1) f)-\sigma^{-1}(p)\right] ; \quad\left(m_{2}=8\right)$

(5) there is a surjective morphism $q: X \rightarrow \Gamma$ onto a smooth curve $\Gamma$ of genus one such that any general fiber $F$ of $q$ is a smooth quadric hypersurface of $\mathbb{P}^{n}$ with $H_{F}=\mathcal{O}_{F}(1)$ and $\mathscr{E}_{F} \cong \mathcal{O}_{F}(1)^{\oplus r}$; moreover, $S$ is the blowing-up $\sigma: S \rightarrow S_{1, e}$ of $S_{1, e} \rightarrow C$ with $e \in\{-1,0\}$ at a point $p$ and $H_{S} \equiv\left[\sigma^{*}\left(2 C_{0}+(e+1) f\right)-\sigma^{-1}(p)\right]$;

(6) $X=\mathbb{P}_{\Sigma}(\mathscr{U})$, where $\Sigma$ is the Jacobian of a smooth curve $\gamma$ of genus 2, $\mathscr{U}$ is an ample vector bundle of rank $n-1$ over $\Sigma$ and $\mathscr{E}=$ $\pi^{*} \mathscr{G} \otimes \xi$, where $\xi$ is the tautological line bundle on $X, \mathscr{G}$ is a vector bundle of rank $r$ on $\Sigma$ and $\pi: X \rightarrow \Sigma$ is the bundle projection; 
moreover, $H_{F}=\mathcal{O}_{F}(t)$ for any fiber $F \cong \mathbb{P}^{n-2}$ of $\pi$ with $t \geq 1$ and $t=1$ if $r<n-2,\left.\pi\right|_{S}: S \rightarrow \Sigma$ is the blowing-up of $\Sigma$ at a point $p$ and $H_{S}=\left.\pi\right|_{S} ^{*} \gamma-\left.\pi\right|_{S} ^{-1}(p)$, looking at the curve $\gamma$ as embedded in its Jacobian.

$\left(m_{2}=6\right)$

Proof. It follows from Table 1 that $\delta=m_{2}-d \geq 3$, with equality if and only if $\left(S, H_{S}\right)=\left(\mathbb{P}^{2}, \mathcal{O}_{\mathbb{P}^{2}}(3)\right)$. By [21, Theorem 4 and Remark in Sec. 2], this pair leads to the first assertion in the statement.

So we continue supposing that $m_{2}-d \geq 4$. Now, assume that equality holds. Taking into account the pairs $\left(S, H_{S}\right)$ in Table 1 , we see that condition $g \leq 1$ forces $\left(S, H_{S}\right)$ to be either $\left(\mathbb{P}^{1} \times \mathbb{P}^{1}, \mathcal{O}(2,2)\right)$ or $\left(S_{0,1},-K_{S_{0,1}}\right)$. In both cases $\left(S, H_{S}\right)$ is a del Pezzo pair, but [21, Theorem 4 and Remark at the end of §2] shows that only the former case lifts to the vector bundle setting giving rise to (1) and (2) in the statement. Next assume $g \geq 2$. If $S$ is not ruled, according to (\#) and the interpretation of $m_{2}$ (see Proposition 2), the equality $m_{2}-d=4$ implies $g=2$ and $e(S)=0$. In this case, by [30, Theorem 4.2], $S$ is a minimal surface, which is either i) an elliptic fibration over $\mathbb{P}^{1}$ with some multiple fibers (see [32]), or ii) an abelian or a bielliptic surface. By [22, Theorem] case i) cannot occur: actually, the fact that $S$ is minimal contradicts [22, Theorem (a)] while the existence of multiple fibers is in contrast with [22, Theorem (b)]. Similarly, case ii) cannot occur since the only minimal surface of Kodaira dimension zero occurring as zero locus of an ample vector bundle is a K3 surface [17, Theorem]. Therefore $S$ is a ruled surface, and then, according to [30, Theorem 4.3] $S$ is a $\mathbb{P}^{1}$-bundle over an elliptic curve; moreover, $g=2$ and one of the following cases holds:

(a) $S:=S_{1,-1}$ and $H_{S} \equiv\left[3 C_{0}-f\right]$;

(b) $S:=S_{1, e}$ with $e \in\{-1,0\}$ and $H_{S} \equiv\left[2 C_{0}+(e+1) f\right]$.

Since $S$ is an irrational $\mathbb{P}^{1}$-bundle, we can use $[20$, Theorem] to conclude that $X$ is a $\mathbb{P}^{n-1}$-bundle over a smooth curve $B$ and $\mathscr{F}_{F}=\mathcal{O}_{\mathbb{P}^{n-1}}(1)^{\oplus(n-2)}$ for every fiber $F$ of the bundle projection $\pi: X \rightarrow B$. This implies that $\left.\pi\right|_{S}$ is the bundle projection of $S, f$ being a line in $F$, hence $B$ is the elliptic base curve of $S$. Moreover, we see that if $r<n-2$, then $H_{F}=\mathcal{O}_{\mathbb{P}^{n-1}}(1)$, as a summand of $\mathscr{F}_{F}$, but this is in contradiction with the fact that $1=H f=H_{S} f=2$ or 3 , according to cases (a) and (b). Thus $r=n-2$ and $H_{F}=\mathcal{O}_{\mathbb{P}^{n-1}}(t)$, with $t=2$ or 3 . This gives (3) in the statement.

Finally, assume that $m_{2}-d=5$. If $g \leq 1$, then equality holds and $S$ is the blowing-up of $\mathbb{P}^{2}$ at two points with $H_{S}=-K_{S}, q=p_{g}=0$ and $e(S)=5$. Since $\left(S, H_{S}\right)$ is a del Pezzo surface, this situation cannot lift to the ample vector bundle setting by [21, Theorem 4 and Remark at the end of $\S 2$ ]. Thus $g \geq 2$. From Table 1 we know that $g=2$ and $\left(S, H_{S}\right)$ is one of the following pairs: 
(i) $\quad \kappa(S) \geq 0, H_{S}^{2}=1$ and $S$ is the blowing-up at a single point of the Jacobian of a smooth curve $C$ of genus 2;

(ii) $S$ is ruled, $q=1$ and $S$ is the blowing-up $\sigma: S \rightarrow S^{\prime}$ at a point $p$ of a $\mathbb{P}^{1}$-bundle $S^{\prime}$ over a smooth curve $B$ of genus 1 and one of the following conditions holds:

(c) $S^{\prime}=S_{1,-1}$ and $H_{S} \equiv\left[\sigma^{*}\left(3 C_{0}-f\right)-\sigma^{-1}(p)\right]$;

(d) $S^{\prime}=S_{1, e}, e=0,-1$ and $H_{S} \equiv\left[\sigma^{*}\left(2 C_{0}+(e+1) f\right)-\sigma^{-1}(p)\right]$.

In case (i), since $S$ is birationally equivalent to an abelian surface, by [17, Theorem] we obtain case (6) in the statement. In case (ii), since $S$ is a nonminimal ruled surface, it follows from [28, Theorem] and Lemmas 4 and 5 that $(X, \mathscr{F}, H)$ is one of the following triplets:

(j) there is a vector bundle $\mathscr{V}$ on a smooth curve $C$ such that $X \cong$ $\mathbb{P}_{C}(\mathscr{V})$ and $\mathscr{F}_{F} \cong \mathcal{O}_{F}(2) \oplus \mathcal{O}_{F}(1)^{\oplus(n-3)}$ for any fiber $F \cong \mathbb{P}^{n-1}$ of $X \rightarrow C$;

(jj) there is a surjective morphism $X \rightarrow C$ onto a smooth curve $C$ such that any general fiber $F$ of $X \rightarrow C$ is a smooth quadric hypersurface of $\mathbb{P}^{n}$ with $\mathscr{\mathscr { F }}_{F} \cong \mathcal{O}_{F}(1)^{\oplus(n-2)}$;

(jjj) there is a vector bundle $\mathscr{U}$ on a smooth surface $\Sigma$ such that $X \cong$ $\mathbb{P}_{\Sigma}(\mathscr{U})$ and $\mathscr{F}_{F} \cong \mathcal{O}_{F}(1)^{\oplus(n-2)}$ for any fiber $F \cong \mathbb{P}^{n-2}$ of $\pi: X \rightarrow \Sigma$.

Write $H_{F}=\mathcal{O}_{F}(t)$ for some positive integer $t$. In cases $(\mathrm{j})$ and $(\mathrm{jj})$, note that the fibration $X \rightarrow C$ restricted to $S$ is the ruling projection $S \rightarrow B$, hence $C \cong B$. Moreover, we have $F_{S}=\sigma^{*} f$ for a general fiber $F$, since $F_{S} \cdot \sigma^{*} f=0$ for any general fiber $f$ of $S^{\prime}, g\left(F_{S}\right)=0$ and $F_{S}^{2}=0$. Since

$$
H_{S} \cdot \sigma^{*} f=H_{S} \cdot F_{S}=H_{F} \cdot S_{F}=H_{F} \cdot c_{n-2}\left(\mathscr{F}_{F}=H_{F} \cdot c_{n-2}\left(\mathscr{F}_{F}\right)=2 t\right.
$$

is even for any fiber $f$ of $S^{\prime}$ and

$$
H_{S} \cdot \sigma^{*} f= \begin{cases}2 & \text { in case }(\mathrm{d}) \\ 3 & \text { in case }(\mathrm{c})\end{cases}
$$

we conclude that only case (d) can occur with $t=1$. This leads to cases (4) and (5) in the statement.

In (jjj), note that case (d) cannot occur by [5, Theorem]. Moreover, in case (c) we have

$$
\left[K_{X}+\operatorname{det}(\mathscr{F} \oplus H)\right]_{S}=K_{S}+H_{S} \equiv\left[\sigma^{*}\left(C_{0}\right)\right] .
$$

Therefore, $K_{X}+\operatorname{det}(\mathscr{F} \oplus H)$ is not ample. So, by [1, Theorem C)] we know that there exist a morphism $s: X \rightarrow W$ expressing $X$ as a smooth projective $n$-fold $W$ blown-up at a finite set $\Gamma \neq \varnothing$ and an ample vector bundle $\mathscr{F}^{\prime}$ on $W$ such that $\mathscr{F} \oplus H=s^{*} \mathscr{F}^{\prime} \otimes\left[-s^{-1}(\Gamma)\right]$ and $K_{W}+\operatorname{det} \mathscr{F}^{\prime}$ is ample. Consider an exceptional divisor $E \cong \mathbb{P}^{n-1}$ of $s$. Since $n-1 \geq 3$, we see that $\pi(E)$ 
is a point of $S^{\prime}$, but this is impossible since any fiber of $\pi$ is a linear $\mathbb{P}^{n-2}$. Therefore, case (c) cannot occur.

REMARK 5. From Theorem 3 we deduce that 1 and 2 are gap values for $\delta$. Thus apart from a short list of triplets $(X, \mathscr{E}, H)$ as in Theorem 3, we have $\delta \geq 6$.

REMARK 6. Let us note here that case (6) in Theorem 3 is effective. Recall that this case comes from case $(13)$ of $[28$, Theorem]. Let $(C, o)$ be a pointed smooth curve of genus 2, and on the Jacobian $J(C)$ of $C$ consider the Jacobian bundle $\mathscr{E}_{r}(C, o)$ of rank $r$, as in [7, (2.18)]. Set $X:=\mathbb{P}\left(\mathscr{E}_{n-1}(C, o)\right)$; then $X$ is a $\mathbb{P}^{n-2}$-bundle over the smooth surface $J(C)$. Recall that $X$ can be identified with $C^{(n)}$, the $n$-fold symmetric product of $C$, the bundle projection $\pi: C^{(n)} \rightarrow J(C)$ being given by the mapping $\left(x_{1}, \ldots, x_{n}\right) \mapsto\left[x_{1}+\cdots+x_{n}-n o\right]$. Let $H$ be the tautological line bundle on $X ; H$ is ample. Moreover, as shown in $[7,(2.18)]$ there is a section of $H$ whose zero locus is $\mathbb{P}\left(\mathscr{E}_{n-2}(C, o)\right)$, which can be identified with $C^{(n-1)}$. By induction, we thus see that $S:=C^{(2)}$ is the zero locus of a section of the ample vector bundle $H^{\oplus(n-2)}$. Thus the triplet $\left(X, \mathscr{E}:=H^{\oplus(n-2)}, H\right)$ provides an example as in case (6) of Theorem 3. Note also that $\left.\pi\right|_{S}: S \rightarrow J(C)$ is just the contraction of the unique (-1)-line of $\left(S, H_{S}\right)$ corresponding to the canonical $g_{2}^{1}$ of $C$.

To avoid long lists repeating several triplets we already met, in the next statement, as well as in Section 5, we simply denote by

$\mathscr{A}$ : the class consisting of the five triplets appearing in Theorem 2;

$\mathscr{B}$ : the class consisting of the first three triplets occurring in Theorem 3, namely, $\quad\left(\mathbb{P}^{n}, \mathcal{O}_{\mathbb{P}^{n}}(1)^{\oplus(n-2)}, \mathcal{O}_{\mathbb{P}^{n}}(3)\right), \quad\left(\mathbb{P}^{n}, \mathcal{O}_{\mathbb{P}^{n}}(2) \oplus \mathcal{O}_{\mathbb{P}^{n}}(1)^{\oplus(n-3)}\right.$, $\left.\mathcal{O}_{\mathbb{P}^{n}}(2)\right)$, and $\left(\mathbb{Q}^{n}, \mathcal{O}_{\mathbb{Q}^{n}}(1)^{\oplus(n-2)}, \mathcal{O}_{\mathbb{Q}^{n}}(2)\right)$.

Thus by Theorems 2 and 3 , combined with Table 1 and the fact that $m_{2}=$ $\delta+d \geq \delta+1$, we have the following consequence.

Corollary 1. Let $(X, \mathscr{E}, H)$ be as in $(\diamond)$. Then

$$
m_{2} \leq 6
$$

if and only if either $(X, \mathscr{E}, H) \in \mathscr{A}\left(m_{2}=0,3,2,2, d \leq 6\right)$, or $(X, \mathscr{E}, H)$ is as in case (6) of Theorem $3\left(m_{2}=6\right)$.

As a consequence of Corollary 1 , we have $m_{2} \geq 7$ apart from a short list of triplets $(X, \mathscr{E}, H)$.

Finally, in line with Corollary 1, we further show that also 7-9 are gap values for $m_{2}$, provided that $S$ is not ruled.

Proposition 3. Let $(X, \mathscr{E}, H)$ be as in $(\diamond)$ and suppose that $S$ is not a ruled surface. If $m_{2} \geq 7$, then $m_{2} \geq 10$. 
Proof. Since $m_{2} \geq 7$ and $S$ is not ruled, we see from Table 1 (where $m$ is now our $m_{2}$ ) that $m_{2} \geq 9$ and equality implies that $S$ is a minimal elliptic surface, $\chi\left(\mathcal{O}_{S}\right)=0, g=3$ and $H_{S}^{2}=1$. In this case, it follows from [22, Theorem(b)] that $X$ is endowed with a morphism $\varphi: X \rightarrow B$ onto a smooth curve $B$ inducing on $S$ the elliptic fibration and $f:=\left.\varphi\right|_{S}: S \rightarrow B$ has no multiple fibres. Thus by $[2,(12.1)$ and (12.2) in Chapter V, pp. 161-162] we deduce that $K_{S}=f^{*}\left(K_{B}\right) \equiv(2 g(B)-2) F$ since deg $f_{* 1}\left(\mathcal{O}_{S}\right)^{\vee}=\chi\left(\mathcal{O}_{S}\right)=0$, where $F$ is a fiber of $f$, but this gives the numerical contradiction

$$
2(g(B)-1) F H_{S}=K_{S} H_{S}=2 g-2-H_{S}^{2}=4-1=3 .
$$

As a consequence of Corollary 1 and Proposition 3, when $S$ is not a ruled surface, we conclude that $m_{2} \geq 10$ apart from a short list of triplets. As to case $m_{2}=10$, one can show that if $\kappa(S)=0$, then $r=n-2, X=$ $\mathbb{P}_{\Sigma}(\mathscr{U})$, for an ample vector bundle $\mathscr{U}$ of rank $n-1$ on a smooth minimal surface $\Sigma$, and $\mathscr{E}=\pi^{*} \mathscr{G} \otimes \xi$, where $\xi$ is the tautological line bundle on $X, \mathscr{G}$ is a vector bundle of rank $n-2$ on $\Sigma$ and $\pi: X \rightarrow \Sigma$ is the bundle projection; furthermore, $H_{F}=\mathcal{O}_{F}(3)$ for any fiber $F \cong \mathbb{P}^{n-2}$ of $\pi,\left.\pi\right|_{S}: S \rightarrow \Sigma$ is the blowing-up of $\Sigma$ at a point $p, e(S)=1$ and $\Sigma$ is either an abelian or a bielliptic surface with $H_{S}=\left.\pi\right|_{S} ^{*} L_{0}-\left.3 \pi\right|_{S} ^{-1}(p)$ and $L_{0}^{2}=10$. We omit the proof for shortness.

\section{Lower bounds for $\delta$ in terms of $g$}

In this section, we will compare $\delta=m_{2}-d$ with the sectional genus $g$ of the polarized surface $\left(S, H_{S}\right)$. A first result is given by the following

Proposition 4. Let $(X, \mathscr{E}, H)$ be as in $(\diamond)$. Assume that $\delta>0$ and $g \geq 2$. Then

$$
\delta \geq 2 g
$$

and equality holds if and only if $r=n-2, X$ is a $\mathbb{P}^{n-1}$-bundle over a smooth curve $B$ and $\mathscr{E}_{F} \cong \mathcal{O}_{F}(1)^{\oplus(n-2)}$ for every fiber $F \cong \mathbb{P}^{n-1}$ of the bundle projection $\pi: X \rightarrow B$. In particular, $S=S_{q, e}$ is a $\mathbb{P}^{1}$-bundle over $B$ via $\left.\pi\right|_{S}$. Moreover, either

(i) $H_{F}=\mathcal{O}_{F}(3), q=1, e=-1, g=2$ and $H_{S} \equiv\left[3 C_{0}-f\right]$, or

(ii) $H_{F}=\mathcal{O}_{F}(2), g=2 q>0, e \leq 0$ and $H_{S} \equiv\left[2 C_{0}+(e+1) f\right]$.

Proof. By [30, Proposition 3.2], we know that $\delta \geq 2 g$, equality holding if and only if one of the following cases occurs:

(1) $S=S_{1,-1}, g=2$ and $H_{S} \equiv\left[3 C_{0}-f\right]$;

(2) $S=S_{q, e}$ with $e \leq 0, g=2 q>0$ and $H_{S} \equiv\left[2 C_{0}+(e+1) f\right]$; 
(3) $S$ is a minimal surface endowed with an elliptic fibration $S \rightarrow \mathbb{P}^{1}$, $q=1, p_{g}=0, g=2$ and $H_{S}^{2}=1$;

(4) $S$ is a minimal nonruled surface with $g=2$.

Note that in case (3), from [4, Theorem 1.5] it follows that $S$ has multiple fibers, but this contradicts [22, Theorem]. Moreover, also case (4) cannot occur by [17, Theorem] since $S$ is minimal and not a K3 surface. Finally, by [20, Theorem] in cases (1) and (2) we conclude that $X$ is a $\mathbb{P}^{n-1}$-bundle over a smooth curve $B$ and $\mathscr{F}_{F} \cong \mathcal{O}_{F}(1)^{\oplus(n-2)}$ for every fiber $F \cong \mathbb{P}^{n-1}$ of the bundle projection $\pi: X \rightarrow B$. Note that $F_{S}=f$ for any fiber $F$ of $\pi$ and that the restriction $\left.\pi\right|_{S}: S \rightarrow B$ of $\pi$ to $S$ gives the bundle projection on $S$. Moreover, we have $H_{F}=\mathcal{O}_{F}(b)$ with $b=3,2$ according to cases (1) and (2) respectively. This shows that necessarily $r=n-2$.

Now, we lift the results of Theorem 1 and Proposition 1 to the ample vector bundle setting.

As a consequence of Theorem 1, we can obtain the following

THEOREM 4. Let $(X, \mathscr{E}, H)$ be as in $(\diamond)$.

(A) If $\delta=2 g+1$, then either $(\delta, g)=(3,1),(5,2)$ and the triplets $(X, \mathscr{E}, H)$ fit into all the possibilities of Theorem 3 for $\delta=3$ and 5 , or $(X, \mathscr{E}, H)$ is one of the following triplets:

(i) $X \cong \mathbb{P}_{C}(\mathscr{V})$, where $\mathscr{V}$ is a vector bundle of rank $n$ on a smooth curve $C, \mathscr{E}_{F} \cong \mathcal{O}_{F}(2) \oplus \mathcal{O}_{F}(1)^{\oplus(r-1)}$ and $H_{F}=\mathcal{O}_{F}(1)$, for any fiber $F \cong \mathbb{P}^{n-1}$ of the bundle projection $X \rightarrow C$;

(ii) there is a surjective morphism $X \rightarrow \Gamma$ onto a smooth curve $\Gamma$ whose general fiber $F$ is a smooth quadric hypersurface of $\mathbb{P}^{n}$ such that $\mathscr{E}_{F} \cong \mathcal{O}_{F}(1)^{\oplus r}$ and $H_{F}=\mathcal{O}_{F}(1)$.

Moreover, in both cases, $S$ is the blowing-up $\sigma: S \rightarrow S_{q, e}$ of a surface $S_{q, e}$ at a point $p, H_{S} \equiv\left[\sigma^{*}\left(2 C_{0}+(e+1) f\right)-\sigma^{-1}(p)\right]$ and $g=$ $2 q \geq 4$.

(B) If $\delta=2 g+2$ and $g \geq 4$, then we have the following possibilities:

(B1) $r=n-2, X$ is a $\mathbb{P}^{n-1}$-bundle over a smooth curve $B$ and $\mathscr{E}_{F} \cong$ $\mathcal{O}_{F}(1)^{\oplus(n-2)}$ for every fiber $F \cong \mathbb{P}^{n-1}$ of the bundle projection $\pi: X \rightarrow B$. Moreover, either

(j) $H_{F}=\mathcal{O}_{F}(3), S=S_{2,-1}, g=5$, and $H_{S} \equiv\left[3 C_{0}-f\right]$, or

(jj) $H_{F}=\mathcal{O}_{F}(2), S=S_{q, e}$ with $q \geq 2, e \leq 0, g=2 q+1$ and $H_{S} \equiv\left[2 C_{0}+(e+2) f\right]$;

(B2) $(X, \mathscr{E}, H)$ is as in (i) and (ii) of (A), and in both cases, $S$ is the blowing-up $\sigma: S \rightarrow S_{q, e}$ of a surface $S_{q, e}$ at two points $p_{1}, p_{2}$, lying on distinct fibers, $H_{S} \equiv\left[\sigma^{*}\left(2 C_{0}+(e+1) f\right)-\sigma^{-1}\left(p_{1}\right)-\right.$ $\left.\sigma^{-1}\left(p_{2}\right)\right]$ and $g=2 q$. 
Proof. (A) If $g \leq 3$ then $\delta=2 g+1 \leq 7$ and from Table 1 we conclude that only $(\delta, g)=(3,1),(5,2)$ satisfy the current assumption, i.e. the triplets $(X, \mathscr{E}, H)$ fit into all the possibilities of Theorem 3 for $\delta=3$ and 5. So we can assume $g \geq 4$. By Theorem 1 we deduce that $g=2 q \geq 4, S$ is the blowingup $\sigma: S \rightarrow S_{q, e}$ of a surface $S_{q, e}$ at a point $p$ and $H_{S} \equiv\left[\sigma^{*}\left(2 C_{0}+(e+1) f\right)-\right.$ $\left.\sigma^{-1}(p)\right]$. Having in mind [5, Theorem] and by arguing as in the proof of Theorem 3, we can easily deduce cases (i) and (ii) of the statement.

(B) Since $\delta=2 g+2$, from Theorem 1 it follows that $\left(S, H_{S}\right)$ is one of the following three polarized surfaces:

(a) $S=S_{2,-1}, g=5, H_{S} \equiv\left[3 C_{0}-f\right]$ and $H_{S}^{2}=3$;

(b) $S=S_{q, e}$ with $q \geq 2, g=2 q+1, H_{S} \equiv\left[2 C_{0}+(e+2) f\right]$ and $H_{S}^{2}=8$;

(c) $S$ is the blowing-up $\sigma: S \rightarrow S_{q, e}$ of $S_{q, e}$ at two points $p_{1}, p_{2}$, lying on distinct fibers, $H_{S} \equiv\left[\sigma^{*}\left(2 C_{0}+(e+1) f\right)-\sigma^{-1}\left(p_{1}\right)-\sigma^{-1}\left(p_{2}\right)\right], \quad g=$ $2 q \geq 4$ and $H_{S}^{2}=2$.

If $\left(S, H_{S}\right)$ is as in (a) and (b), then by arguing as in cases (1) and (2) of the proof of Proposition 4, we obtain (B1) in the statement. In case (c), recalling [5, Theorem] and reasoning as in the proof of Theorem 3, we get (B2) in the statement.

THEOREM 5. Let $(X, \mathscr{E}, H)$ be as in $(\diamond)$ and suppose that $S$ is not a ruled surface. Then $\delta \geq 2 g+d$.

Proof. Simply note that cases (1) and (2) of Proposition 1 cannot ascend to the ample vector bundle setting due to [17, Theorem] and [22, Theorem]. Actually, in the former case $S$ is a minimal surface of Kodaira dimension zero, while in the latter $S$ is an elliptic surface with multiple fibers.

\section{When $H_{S}$ is ample and spanned or very ample}

In this Section, we revisit all the above results in the ample and spanned (Subsection 4.1) or very ample (Subsection 4.2) settings and we improve some of them.

5.1. $H_{S}$ is an ample and spanned line bundle. First of all, note that if $\delta \leq 3$, then the triplets $(X, \mathscr{E}, H)$ are as in Theorems 2 and 3. Thus, assume that $\delta>3$.

THEOREM 6. Let $(X, \mathscr{E}, H)$ be as in $(\diamond)$, suppose that condition (S) holds, and let $\delta \geq 4$. Then

$$
\delta \geq 9
$$

except in the following cases: 
(1) $\delta=4$ and $(X, \mathscr{E}, H)$ is either as in cases (1) and (2) of Theorem $3\left(m_{2}=12\right)$, or $r=n-2, X$ is a $\mathbb{P}^{n-1}$-bundle over a smooth curve $B$ of genus $1, \mathscr{E}_{F}=\mathcal{O}_{\mathbb{P}^{n-1}}(1)^{\oplus(n-2)}$ and $H_{F}=\mathcal{O}_{\mathbb{P}^{n-1}}(2)$ for every fiber $F=\mathbb{P}^{n-1}$ of the bundle projection $X \rightarrow B$ and $\left(S, H_{S}\right) \cong\left(S_{1,-1},\left[2 C_{0}\right]\right)$

$\quad\left(m_{2}=8\right)$;

(2) $\delta=6 \quad$ and $\quad(X, \mathscr{E}, H) \cong\left(\mathbb{P}^{2} \times \mathbb{P}^{2}, \mathcal{O}_{\mathbb{P}^{2} \times \mathbb{P}^{2}}(1,1)^{\oplus 2}, \mathcal{O}_{\mathbb{P}^{2} \times \mathbb{P}^{2}}(1,1)\right)$; $\left(m_{2}=12\right)$

(3) $\delta=7$ and we have either $(X, \mathscr{E}, H) \cong\left(\mathbb{Q}^{4}, \mathscr{S} \otimes \mathcal{O}_{\mathbb{Q}^{4}}(2), \mathcal{O}_{\mathbb{Q}^{4}}(1)\right)$, where $\mathscr{S}$ is a spinor bundle on $\mathbb{Q}^{4}$, or $X$ is a linear section of the Grassmannian variety $\mathbb{G}(1,4) \subset \mathbb{P}^{9}$ and $(\mathscr{E}, H) \cong\left(L^{\oplus r}, L\right)$, where $L$ is the ample generator of $\operatorname{Pic}(X)$;

$\left(m_{2}=12\right)$

(4) $\delta=8$ and $(X, \mathscr{E}, H)$ is one of the following triplets:

(a) $\quad\left(\mathbb{P}^{n}, \mathcal{O}_{\mathbb{P}^{n}}(2)^{\oplus 2} \oplus \mathcal{O}_{\mathbb{P}^{n}}(1)^{\oplus(r-2)}, \mathcal{O}_{\mathbb{P}^{n}}(1)\right) ; \quad\left(m_{2}=12\right)$

(b) $\quad\left(\mathbb{Q}^{n}, \mathcal{O}_{\mathbb{Q}^{n}}(2) \oplus \mathcal{O}_{\mathbb{Q}^{n}}(1)^{\oplus(r-1)}, \mathcal{O}_{\mathbb{Q}^{n}}(1)\right) ; \quad\left(m_{2}=12\right)$

(c) $X$ is a complete intersection of two quadric hypersurfaces of $\mathbb{P}^{n+2}$ and $(\mathscr{E}, H) \cong\left(L^{\oplus r}, L\right)$, where $L$ is the ample generator of $\operatorname{Pic}(X)$;

$\left(m_{2}=12\right)$

(d) $r=n-2$ and there is a vector bundle $\mathscr{V}$ on a smooth curve $C$ of genus $q \leq 2$ such that $X \cong \mathbb{P}_{C}(\mathscr{V}), H_{F}=\mathcal{O}_{F}(2)$ and $\mathscr{E}_{F} \cong$ $\mathcal{O}_{F}(1)^{\oplus(n-2)}$ for any fiber $F \cong \mathbb{P}^{n-1}$ of $X \rightarrow C$; moreover, $\left(S, H_{S}\right)$ is, up to numerical equivalence, one of the following pairs:

$\left(d_{1}\right) \quad\left(S_{0, e},\left[2 C_{0}+(e+3) f\right]\right)$, with $e \leq 2 ; \quad\left(m_{2}=20\right)$

$\left(d_{2}\right) \quad\left(S_{1, e},\left[2 C_{0}+(e+2) f\right]\right)$, with $e \leq 0 ; \quad\left(m_{2}=16\right)$

$\left(d_{3}\right) \quad\left(S_{2, e},\left[2 C_{0}+(e+1) f\right]\right)$, with $e \leq-1 . \quad\left(m_{2}=12\right)$

Proof. If $\delta=4$, then from Theorem 3 it follows case (1) of the statement. Actually, by [23, Theorem (3.1)] the remaining possibilities in Theorem 3 (3) cannot occur, $H_{S}$ being spanned. If $\delta=5$, then by Lemma 2 and Table 1 we deduce that the only possible cases for $\left(S, H_{S}\right)$ are N. 12 and 15 of Table 1. The former case does not lift to the vector bundle setting by [21, Theorem 4 and Remark in §2] and the latest one cannot occur since $g=2$ and $H_{S}$ is required to be ample and spanned (see [23, Theorem (3.1)]). This shows that $\delta=5$ cannot occur. If $\delta=6,7$ we have $g=1$ or 2 , by Table 1 . If $g=1$, from Lemma 2 and [21, Theorem 4 and Remark in §2] we obtain immediately cases (2) and (3) of the statement. On the other hand it cannot be $g=2$ because $H_{S}$ is ample and spanned: actually, in cases N. 17, 18 and 20 of Table $1, S$ is not a minimal surface and this is not compatible with [23, Theorem (3.1)] again. Suppose now that $\delta=8$. First of all, assume that $\kappa(S) \geq 0$. Then $\left(S, H_{S}\right)$ is as in cases N. 22, 23 and 24 of Table 1. Since $H_{S}$ is ample and spanned and $g=3$ in all cases, by [18, Table I, p. 268] we see that N. 22 and 23 cannot occur and that in N. 24 the surface $S$ is a minimal elliptic 
fibration with multiple fibers, but this situation does not lift to the vector bundle setting by [22, Theorem]. Finally, suppose that $\kappa(S)=-\infty$, i.e. $S$ is a ruled surface. From Table 1 we deduce that either

(i) $H_{S}^{2}=4, g=1, e(S)=8$,

or $\left(S, H_{S}\right)$ is, up to numerical equivalence, one of the following pairs:

(ii) $\left(S_{0, e},\left[2 C_{0}+(3+e) f\right]\right)$ with $e=0,1,2, H_{S}^{2}=12, g=2, e(S)=4$;

(iii) $\left(S_{1, e},\left[2 C_{0}+(2+e) f\right]\right)$ with $e=-1,0,1, H_{S}^{2}=8, g=3, e(S)=0$;

(iv) $\left(S_{1,0},\left[3 C_{0}+f\right]\right)$ with $H_{S}^{2}=6, g=3, e(S)=0$;

(v) $\left(S_{1,-1},\left[5 C_{0}-2 f\right]\right)$ with $H_{S}^{2}=5, g=3, e(S)=0$;

(vi) $\left(S_{2, e},\left[2 C_{0}+(e+1) f\right]\right)$ with $-2 \leq e \leq 0, H_{S}^{2}=4, g=4, e(S)=-4$. Note that (iv) cannot occur since in this case $H_{S} \cdot C_{0}=1$ with $g\left(C_{0}\right)=1$ implies that $H_{S}$ is not spanned. Moreover, from [18, Table II, p. 268] it follows that also case $(\mathrm{v})$ is not possible since $0=e(S)=12(1-q)-K_{S}^{2}$. In case (i), by [21, Theorem 4 and Remark in $\S 2$ ] we get cases $(a),(b)$ and $(c)$ of the statement. Finally, having in mind that $H_{S}$ is ample and spanned, cases (ii), (iii) and (vi) lead to cases $\left(d_{1}\right),\left(d_{2}\right)$ and $\left(d_{3}\right)$ of the statement by [20].

Corollary 2. Let $(X, \mathscr{E}, H)$ be as in $(\diamond)$ and suppose that condition (S) holds. Then

$$
m_{2} \leq 11
$$

if and only if either $(X, \mathscr{E}, H) \in \mathscr{A}\left(m_{2}=0,3,2,2, d \leq 11\right)$, or $r=n-2, X$ is a $\mathbb{P}^{n-1}$-bundle over a smooth curve $B$ of genus $1, \mathscr{E}_{F}=\mathcal{O}_{\mathbb{P}^{n-1}}(1)^{\oplus(n-2)}$ and $H_{F}=$ $\mathcal{O}_{\mathbb{P}^{n-1}}(2)$ for every fiber $F=\mathbb{P}^{n-1}$ of the bundle projection $X \rightarrow B$ and $\left(S, H_{S}\right) \cong$ $\left(S_{1,-1},\left[2 C_{0}\right]\right) \quad\left(m_{2}=8\right)$.

Proof. Since $m_{2}=\delta+H_{S}^{2}$ and $H_{S}^{2} \geq 3$ unless a few exceptions for the pairs $\left(S, H_{S}\right)$ described in Lemma 2 , the result follows from Theorems 2, 3 and 6 .

Corollary 3. Let $(X, \mathscr{E}, H)$ be as in $(\diamond)$ and suppose that $(\mathrm{S})$ holds. Then

$$
\delta \geq 2 g+3
$$

unless either $g \geq 4$ and $(X, \mathscr{E}, H)$ is as in Proposition 4 and Theorem 4 , or $g \leq 3$ and one of the following cases occurs:

(1) $(X, \mathscr{E}, H) \in \mathscr{A} \cup \mathscr{B} \quad\left(m_{2}=0,3,2,2, d, 12,12,12\right)$;

(2) $\quad r=n-2, X$ is a $\mathbb{P}^{n-1}$-bundle over a smooth curve $B$ of genus $1, \mathscr{E}_{F}=$ $\mathcal{O}_{\mathbb{P}^{n-1}}(1)^{\oplus(n-2)}, H_{F}=\mathcal{O}_{\mathbb{P}^{n-1}}(2)$ for every fiber $F=\mathbb{P}^{n-1}$ of the bundle projection $X \rightarrow B$ and $\left(S, H_{S}\right) \cong\left(S_{1,-1},\left[2 C_{0}\right]\right) \quad\left(m_{2}=8\right)$; 
(3) $r=n-2$ and there is a vector bundle $\mathscr{V}$ on a smooth curve $C$ of genus $q=1$ such that $X \cong \mathbb{P}_{C}(\mathscr{V}), H_{F}=\mathcal{O}_{F}(2), \mathscr{E}_{F} \cong \mathcal{O}_{F}(1)^{\oplus(n-2)}$ for any fiber $F \cong \mathbb{P}^{n-1}$ of $X \rightarrow C$ and $\left(S, H_{S}\right)$ is, up to numerical equivalence, $\left(S_{1, e},\left[2 C_{0}+(e+2) f\right]\right)$ with $e \in\{-1,0\}$.

$\left(m_{2}=16\right)$

Proof. Let $\delta \leq 2 g+2$. If $g \leq 3$, then $\delta \leq 8$ and the assertion follows from Theorems 2, 3 and 6. If $g \geq 4$, then Proposition 4 and Theorem 4 apply.

When $S$ is not a ruled surface, we have the following two results.

Proposition 5. Let $(X, \mathscr{E}, H)$ be as in $(\diamond)$ and suppose that $(\mathrm{S})$ holds. If $S$ is not a ruled surface, then $\delta \geq 2 g+5$.

Proof. Recall that $\delta=m_{2}-d$ and assume, by contradiction, that $\delta \leq$ $2 g+4$. If $d \leq 2$, then from Lemma 2 it follows that $d=2, g=b-1$ and $e(S)=2\left(2 b^{2}-3 b+3\right)$ for some integer $b \geq 3, S$ being not a ruled surface. Then

$$
\delta=e(S)+4(g-1)=2 g+\left[2 b-6+2\left(2 b^{2}-3 b+3\right)\right] \geq 2 g+24,
$$

which is impossible. Moreover, if $g \leq 2$ then $g=2$ and by [23, Theorem (3.1)] the only possibility for $S$ is to be a K3 surface, in which case however, $\delta=$ $e(S)+4(g-1)=2 g+e(S)+2 g-4=2 g+24$. So we can assume that $d \geq 3$ and $g \geq 3$. Note that by Theorem 5 cases $\delta=2 g+1,2 g+2$ do not occur. Therefore, it is enough to show that also cases $\delta=2 g+3,2 g+4$ cannot occur.

First suppose that $\delta=2 g+3$. Then from (\#) we deduce that $e(S)+2 g=$ 7 and then $(e(S), g)=(1,3)$ since $e(S) \geq 0, S$ being not a ruled surface, and $g \geq 3$. Thus $4=2 g-2=d+H_{S} K_{S} \geq 3+H_{S} K_{S}$, hence $H_{S} K_{S} \leq 1$. It cannot be $H_{S} K_{S}=0$, otherwise, $K_{S}$ would be numerically trivial, due to the ampleness of $H$, but in this case, $S$ could not satisfy $e(S)=1$, in view of the classification. Therefore $H_{S} K_{S}=1, H_{S}^{2}=3$ and by Lemma 1 we see that $\kappa(S)=0$. Moreover, $\left(S, H_{S}\right)$ has $\left(S_{0}, L_{0}\right)$ as simple reduction, where $S_{0}$ is a minimal surface of Kodaira dimension zero with $e\left(S_{0}\right)=0$. So $S_{0}$ is either abelian or bielliptic, and therefore $\chi\left(\mathcal{O}_{S_{0}}\right)=0$. On the other hand, with the same notation as in Lemma $1, H_{S}=\sigma^{*} L_{0}-E, \sigma: S \rightarrow S_{0}$ being the reduction morphism contracting the exceptional curve $E$ at $p \in S_{0}$. We have $h^{0}\left(H_{S}\right)=$ $h^{0}\left(L_{0}\right)-\varepsilon$ where $\varepsilon=0$ or 1 according to whether $p$ is a base point of $\left|L_{0}\right|$ or not. Then, due to the spannedness of $H_{S}$, by the Riemann-Roch theorem and the Kodaira vanishing theorem we get

$$
3 \leq h^{0}\left(H_{S}\right)=h^{0}\left(L_{0}\right)-\varepsilon=\chi\left(\mathcal{O}_{S_{0}}\right)+\frac{1}{2} L_{0}^{2}-\varepsilon=\chi\left(\mathcal{O}_{S_{0}}\right)+2-\varepsilon .
$$

This gives $0=\chi\left(\mathcal{O}_{S_{0}}\right) \geq 1+\varepsilon \geq 1$, a contradiction. 
Finally, suppose that $\delta=2 g+4$. By arguing as in case $\delta=2 g+3$, we get only two possibilities for $(e(S), g)$, namely,

a) $(2,3)$, or

b) $(0,4)$.

In case a), by using (\#) again, we see that $e(S)+2(g-3)=2$, hence $\left(H_{S}^{2}, H_{S} K_{S}\right)$ is either $(3,1)$ or $(4,0)$, by genus formula. Both possibilities rule out. Actually, in the latter case $K_{S}$ would be numerically trivial, but this cannot occur for $e(S)=2$. In the former case, $S$ could be minimal with $\kappa(S)=1$, but then $K_{S}^{2}=0$, which contradicts condition $e(S)=2$ in view of Noether's formula. So $S$ is not minimal. Thus Lemma 1 implies that $\kappa(S)=$ 0 and then $e(S)=2$ says that $S$ is an abelian or a bielliptic surface blown-up at two points. Then $K_{S} \equiv E$ where $E$ consists of two irreducible curves, hence $1=H_{S} K_{S}=H_{S} E \geq 2$, a contradiction.

Now consider case b). By using the facts that $d \geq 3$ and $H_{S} K_{S} \geq 0$, we get for $\left(d, H_{S} K_{S}\right)$ the following list of possible values: $(6,0),(5,1),(4,2)$, $(3,3)$. If $H_{S} K_{S}=0$ (first case), recalling that $e(S)=0$ we conclude that $S$ is either an abelian or a bielliptic surface. Both cases do not ascend the ample vector bundle setting due to [17, Theorem]. If $H_{S} K_{S}=1$ (second case), then Lemma 1 ii) implies that $\kappa(S)=0$ and then $e(S)=0$ allows us to conclude that $S$ is an abelian or a bielliptic surface; but then we get $0=H_{S} K_{S}=1$, a contradiction. Next let us deal with the third and the fourth cases at the same time. Since $e(S)=0$ we have that either i) $S$ is an abelian or a bielliptic surface, or ii) $S$ is a minimal elliptic fibration. In subcase i) $K_{S}$ is numerically trivial, hence $0=H_{S} K_{S}=2$ or 3 , a contradiction. In subcase ii) we have $K_{S}^{2}=0$. This combined with the fact that $e(S)=0$ implies $\chi\left(\mathcal{O}_{S}\right)=0$, by Noether's formula. Now use [24, table in Proposition 4.4 and Proposition 1.4]. For $d=3$, since $S$ is a minimal elliptic surface, [24, Proposition 1.4, case (1.4.2), (i)] shows that $\chi\left(\mathcal{O}_{S}\right)=3$, a contradiction. On the other hand, for $d=4$, since $\chi\left(\mathcal{O}_{S}\right)=0$, [24, table in Proposition 4.4] shows that necessarily the elliptic fibration of $S$ has some multiple fibers. Therefore this case does not ascend to the ample vector bundle setting in view of [22, Theorem].

THEOREM 7. Let $(X, \mathscr{E}, H)$ be as in $(\diamond)$ and suppose that $(\mathrm{S})$ holds. If $S$ is not a ruled surface and $\delta=2 g+5$, then $X=\mathbb{P}_{S_{0}}(\mathscr{V})$, where $\mathscr{V}$ is a vector bundle of rank $(n-1)$ over a smooth minimal surface $S_{0}$, which is either abelian or bielliptic; moreover, $r=n-2$ and $\mathscr{E}=\pi^{*} \mathscr{G} \otimes \xi$, where $\xi$ is the tautological line bundle on $X, \mathscr{G}$ is a vector bundle of rank $n-2$ on $S_{0}$ and $\pi: X \rightarrow S_{0}$ is the bundle projection; furthermore, $\left.\pi\right|_{S}: S \rightarrow S_{0}$ is a birational morphism expressing $S$ as $S_{0}$ blown-up at a single point, say $p . \quad$ Finally, $H=2 \xi+\pi^{*}(A-2(\operatorname{det} \mathscr{V}+$ $\operatorname{det} \mathscr{G})$ ), where $A$ is an ample and spanned line bundle on $S_{0}$ with $A^{2}=8$ and $p$ belongs to its second jumping set $\mathscr{J}_{2}\left(S_{0}, A\right)$. 
For the definition of the jumping sets of an ample and spanned line bundle we refer to [25].

Proof. In view of $(\#)$, the relation $\delta=m_{2}-d=2 g+5$ converts into

$$
e(S)+2(g-3)=3 .
$$

We have $e(S) \geq 0$ by the Castelnuovo-de Franchis Theorem [3, Theorem X.4], hence $g \leq 4$. Clearly it cannot be $g \leq 1$, since $S$ is not a ruled surface. Moreover, for $g=2$, the only pair $\left(S, H_{S}\right)$ with $S$ a nonruled surface is the K3 double plane, according to the classification in [23, Theorem 3.1], but in this case $e(S)=24$, which contradicts (3).

Suppose that $g=3$; then $e(S)=3$ by (3). Clearly $H_{S}^{2} \geq 2$ and taking into account Lemma 2 we see that $e(S)=3$ is not compatible with $H_{S}^{2}=2$. Thus the genus formula, combined with the fact that $S$ is not a ruled surface, implies $\left(H_{S}^{2}, H_{S} K_{S}\right)=(4,0)$, or $(3,1)$. In the former case $K_{S}$ is numerically trivial, hence $S$ is a minimal surface with Kodaira dimension $\kappa(S)=0$, but this contradicts $e(S)=3$. In the latter case the Hodge index theorem shows that $K_{S}^{2} \leq 0$. Suppose that $S$ is minimal. Thus $K_{S}^{2}=0$, since $\kappa(S) \geq 0$, but then Noether's formula contradicts $e(S)=3$ again. Therefore $S$ is not minimal. Let $\eta: S \rightarrow S_{0}$ be a birational morphism to the minimal model. We know that $K_{S}=\eta^{*} K_{S_{0}}+E$, where $E$ is an effective divisor contracted by $\eta$ to a finite set. Consider the equality $1=H_{S} K_{S}=H_{S} \eta^{*} K_{S_{0}}+H_{S} E$ : the second summand on the right hand is greater than or equal to the number of blowing-ups $\eta$ factors through; on the other hand, the first one is non-negative and it is zero if and only if $K_{S_{0}}$ is numerically trivial. It follows that $S$ is $S_{0}$ blown-up at a single point, $E$ being the corresponding exceptional curve, and $\kappa(S)=0$. But then $e(S)=e\left(S_{0}\right)+1 \neq 3$, a contradiction. Thus $g=3$ cannot occur as well.

It remains to consider the case $(e(S), g)=(1,4)$. Clearly $H_{S}^{2} \geq 2$ and by Lemma 2 we see that condition $e(S)=1$ is not compatible with $H_{S}^{2}=2$, as before. Thus the genus formula, combined with the fact that $S$ is not a ruled surface, implies $3 \leq H_{S}^{2} \leq 6$. A close inspection of [24] shows that it cannot be $e(S)=1$ if $H_{S}^{2}=3,5$ or 6 . Actually, as observed before, $S$ cannot be of general type, hence it has Kodaira dimension $\kappa(S)=0$ or 1 . According to [24, Proposition 1.4, Lemma 2.1 combined with Proposition 2.3, and Proposition 3.1], we see that condition $e(S)=1$ would be contradicted. So, $H_{S}^{2}=4$. Now, from [24, Proposition 1.6] we easily see that $e(S)=1$ can occur only when $S$ is a 4-tuple cover of $\mathbb{P}^{2}$ via $\left|H_{S}\right|$, i.e. $h^{0}\left(H_{S}\right)=3$. Clearly condition $e(S)=1$ prevents $S$ from being a minimal surface. Thus, if $\kappa(S)=1[24$, Proposition 4.3] would imply that $\left(S, H_{S}\right)$ is obtained by blowing-up a single point on a minimal elliptic surface with $q=0$. Thus $\chi\left(\mathcal{O}_{S}\right) \geq 1$. By Noether's 
formula we have $K_{S}^{2}+1=K_{S}^{2}+e(S) \geq 12$, hence $K_{S}^{2} \geq 11$. But this is not compatible with $\kappa(S)=1$. This check settles all possibilities, except when $\kappa(S)=0$ and $H_{S}^{2}=4$, in which case $H_{S} K_{S}=2$ by genus formula. Let $\eta: S \rightarrow$ $S_{0}$ be a birational morphism from $S$ to its minimal model $S_{0}$. Since $e(S)=1$, $\eta$ is simply the blowing-up at a point $p \in S_{0}$; in particular, we get $e\left(S_{0}\right)=0$, hence the surface $S_{0}$ is either abelian or bielliptic. From $2=K_{S} H_{S}=$ $\left(\eta^{*} K_{S_{0}}+E\right) H_{S}$, where $E=\eta^{-1}(p)$ is the exceptional curve, we see that $H_{S} E=2, K_{S_{0}}$ being numerically trivial; hence $H_{S}=\eta^{*} A-2 E$, where $A$ is an ample line bundle on $S_{0}$, and $4=H_{S}^{2}=A^{2}-4$, i.e. $A^{2}=8$. Thus

$$
h^{0}(A)=\chi\left(\mathcal{O}_{S_{0}}\right)+\frac{1}{2}\left(A^{2}-A K_{S_{0}}\right)=4,
$$

by the Riemann-Roch and the Kodaira vanishing theorems. Moreover, since $A^{2}=8$ it follows from Reider's Theorem [31, Theorem 1] that either $A$ is a spanned line bundle, or there exists an effective divisor $\Gamma$ on $S_{0}$ such that $A \Gamma=1$ and $\Gamma^{2}=0$. But in the latest case $\Gamma$ is an elliptic curve, since $S_{0}$ cannot contain rational curves. Thus its proper transform on $S, \tilde{\Gamma}=\eta^{*} \Gamma-\varepsilon E$ (where $\varepsilon=0$ or 1 ) is also an elliptic curve, and then $H_{S} \tilde{\Gamma}=\left(\eta^{*} A-2 E\right)\left(\eta^{*} \Gamma-\right.$ $\varepsilon E) \leq A \Gamma=1$, contradicting the spannedness of $H_{S}$. Therefore $A$ is an ample and spanned line bundle on $S_{0}$. According to the above, $\left|H_{S}\right|$ is in bijection with the linear system $|A-2 p|$ of divisors in $|A|$ having a double point at $p$. Recalling that $h^{0}\left(H_{S}\right)=3$, this shows that

$$
3=h^{0}\left(H_{S}\right)=h^{0}(A)-\sharp=4-\sharp,
$$

where $\sharp$ stands for the number of linearly independent linear conditions to be imposed on the elements of $|A|$ in order to have a double point at $p$. Therefore $\sharp=1$. This says that $\operatorname{codim}_{|A|}(|A-2 p|)=1$. Thus, the spannedness of $A$ implies that $|A-2 p|=|A-p|$, i.e. the point $p$ is in the second jumping set $\mathscr{J}_{2}\left(S_{0}, A\right)$. Now come back to the ample vector bundle setting. By using [17, Theorem], we conclude that $X$ is as in the statement with $\mathscr{F}=\pi^{*} \mathscr{G} \otimes \xi$, where $\xi$ is the tautological line bundle on $X, \mathscr{G}$ is a vector bundle of rank $n-2$ on $S_{0}$ and $\pi: X \rightarrow S_{0}$ is the bundle projection; moreover, $\left.\pi\right|_{S}: S \rightarrow S_{0}$ is just the birational morphism $\eta$ expressing $S$ as $S_{0}$ blown-up at the single point $p$. Now consider $H$. If $r<n-2$, then $H_{F}$ is a summand of $\mathscr{F}_{F}$, hence $H_{F}=\mathcal{O}_{\mathbb{P}^{n-2}}(1)$. Since $E$ is contained in a fiber $F$ of $\pi$ and is a line with respect to $\xi_{F}$, we get the contradiction $1=\operatorname{deg}\left(H_{F}\right)_{E}=H_{S} E=2$. Therefore $r=n-2$, hence $\mathscr{F}=\mathscr{E}$. Since $H$ is ample we have $H_{F}=t \xi_{F}=\mathcal{O}_{\mathbb{P}^{n-2}}(t)$ for some positive integer $t$, and then we see from the equality $t=\operatorname{deg}\left(H_{F}\right)_{E}=H_{S} E=2$ that $H_{F}=2 \xi_{F}$. So, $H=2 \xi+\pi^{*} \mathscr{M}$ for some line bundle $\mathscr{M}$ on $S_{0}$, which we have to determine. Recall that $H_{S}=\left.\pi\right|_{S} ^{*} A-2 E$, where $A$ is the ample and spanned 
line bundle on $S_{0}$ with $A^{2}=8$ we met before. Now, by adjunction $K_{S}=$ $\left(K_{X}+\operatorname{det} \mathscr{F}\right)_{S}$ and then by the canonical bundle formula we get

$$
\begin{aligned}
K_{S} & =\left(-(n-1) \xi+\pi^{*}\left(K_{S_{0}}+\operatorname{det} \mathscr{V}\right)+(n-2) \xi+\pi^{*} \operatorname{det} \mathscr{G}\right)_{S} \\
& =-\xi_{S}+\left.\pi\right|_{S} ^{*}\left(K_{S_{0}}+\operatorname{det} \mathscr{V}+\operatorname{det} \mathscr{G}\right) .
\end{aligned}
$$

On the other hand, $K_{S}=\left.\pi\right|_{S} ^{*} K_{S_{0}}+E$, which provides the expression of $E$; hence

$$
\begin{aligned}
H_{S} & =\left.\pi\right|_{S} ^{*} A-2 E \\
& =\left.\pi\right|_{S} ^{*} A-2\left(-\xi_{S}+\left.\pi\right|_{S} ^{*}(\operatorname{det} \mathscr{V}+\operatorname{det} \mathscr{G})\right) \\
& =2 \xi_{S}+\left.\pi\right|_{S} ^{*}(A-2(\operatorname{det} \mathscr{V}+\operatorname{det} \mathscr{G})) .
\end{aligned}
$$

Finally, from the injectivity of the restriction homomorphism $\operatorname{Pic}(X) \rightarrow \operatorname{Pic}(S)$ (Lefschetz-Sommese Theorem), we get the expression of $H$ as in the statement.

REMARK 7. i) We want to stress that Theorem 7 is effective. To see this it is enough to modify the example produced in Remark 6, as follows. Let $X$ be the Jacobian bundle $\pi: \mathbb{P}\left(\mathscr{E}_{n-1}(C, o)\right) \rightarrow S_{0}=J(C)$ on the Jacobian of a smooth curve $C$ of genus 2 again, and call $\xi$ the tautological line bundle. Letting $\mathscr{E}=\xi^{\oplus(n-2)}$ and taking $H=2 \xi$, we see that $H$ is ample and spanned [12, Example 5.1], and the triplet $(X, \mathscr{E}, H)$ is as in Theorem 7: here $S=C^{(2)}$ again, but $A$ is the line bundle corresponding to the double of the curve $C$ itself embedded in its Jacobian. Unfortunately, we have no examples with $S_{0}$ a bielliptic surface.

ii) According to the discussion in the first part of the proof we have to stress a gap affecting the proof of [24, Proposition 4.5]. Actually, the equality in the first case of $(4.5 .1)$ of $[24, \mathrm{p}$. 101] holds provided that the point $p$ does not belong to the first jumping set of $L^{\prime}$ (see $[25, \S 1]$ ): to wit, set $\mathscr{J}_{i}:=$ $\mathscr{J}_{i}\left(X^{\prime}, L^{\prime}\right)$, for $i=0,1,2$, where $\mathscr{J}_{0}=X \backslash \mathscr{J}_{1}$; using the same notation as there, if $L^{\prime}$ is spanned then the mentioned equality has to be amended as follows: $h^{0}\left(L^{\prime}\right)=h^{0}(L)+3-i$, where $p \in \mathscr{J}_{i}$. As a consequence, pairs $\left(X^{\prime}, L^{\prime}\right)$ with $X^{\prime}$ an abelian or a bielliptic surface when $p \in \mathscr{J}_{2}$ and with $X^{\prime}$ an Enriques surface when $p \in \mathscr{J}_{1} \backslash \mathscr{J}_{2}$ are not ruled out.

5.2. Revisiting the classical setting. As a consequence of Remark 1, revisiting Theorem 6 and Corollary 2, we obtain the following two results.

Corollary 4. Let $(X, \mathscr{E}, H)$ be as in $(\diamond)$, suppose that condition (VA) holds, and let $\delta \geq 4$. Then

$$
\delta \geq 9
$$

except in the following cases: 
(1) $\delta=4$ and $(X, \mathscr{E}, H)$ is as in cases (1) and (2) of Theorem $3\left(m_{2}=12\right)$;

(2) $\delta=6,7$ and $(X, \mathscr{E}, H)$ is as in cases (2) and (3) of Theorem 6, respectively $\left(m_{2}=12\right)$;

(3) $\delta=8$ and $(X, \mathscr{E}, H)$ is as in cases $(4)(a),(b),(c)\left(m_{2}=12\right)$ and cases $(4)\left(d_{1}\right) \quad\left(m_{2}=20\right)$ and $(4)\left(d_{2}\right)$ with $e=-1$ of Theorem 6 $\left(m_{2}=16\right)$.

Corollary 5. Let $(X, \mathscr{E}, H)$ be as in $(\diamond)$ and suppose that condition (VA) holds. Then

$$
m_{2} \leq 11
$$

if and only if $(X, \mathscr{E}, H) \in \mathscr{A}\left(m_{2}=0,3,2,2, d \leq 11\right)$.

Recently, Fukuma [11] improved a result of the first author, showing the following

Proposition 6. Let $S$ be a smooth surface endowed with a very ample line bundle $L$, and let $d, g, m$ be the degree, the sectional genus and the class of $(S, L)$. Suppose that $m>d$ and $g \geq 2$. Then $m \geq d+2 g+2$ and equality holds if and only if $(S, L)=\left(S_{1,-1},\left[2 C_{0}+f\right]\right)$ (in which case $d=8, g=3$ ).

Note that the above pair $(S, L)$ corresponds to N. 26 with $e=-1$ in Table 1. In particular, it fits into case $(\mathrm{B})(\gamma)$ with $(m-d, g)=(8,3)$ of Theorem 1 . Coming back to triplets as in $(\diamond)$, observe that for $g:=g\left(S, H_{S}\right) \leq 1$, condition $\delta \leq 2 g+2$ simply means $\delta \leq 4$. Then taking into account Theorems 2,3 and 6 , the very ampleness of $H_{S}$ implies that $(X, \mathscr{E}, H) \in \mathscr{A} \cup \mathscr{B}$. Thus we can assume that $g \geq 2$ and so Proposition 6 can be easily lifted to the ample vector bundle setting, as follows.

Proposition 7. Let $(X, \mathscr{E}, H)$ be as in $(\diamond)$ and suppose that (VA) holds. Assume $g:=g\left(S, H_{S}\right) \geq 2$ and $\delta>0$. Then

$$
\delta \geq 2 g+2
$$

and equality holds if and only if $r=n-2, X$ is a $\mathbb{P}^{n-1}$-bundle over an elliptic curve $B, \mathscr{E}_{F}=\mathcal{O}_{\mathbb{P}^{n-1}}(1)^{\oplus(n-2)}, H_{F}=\mathcal{O}_{\mathbb{P}^{n-1}}(2)$ for every fiber $F \cong \mathbb{P}^{n-1}$ of the projection $X \rightarrow B$, and $\left(S, H_{S}\right)$ is the pair $(S, L)$ described in Proposition 6 .

Proof. Note that $\left(S, H_{S}\right)$ satisfies all the assumptions of Proposition 6 with $L=H_{S}$. This implies the claimed inequality. Now suppose that equality holds; then $\left(S, H_{S}\right)$ is the pair $(S, L)$ described in Proposition 6. Set $\mathscr{F}=$ $\mathscr{E} \oplus H^{\oplus(n-r-2)}$. Since $S$ is a $\mathbb{P}^{1}$-bundle over an elliptic curve, say $B$, we can conclude by [20] that $X$ is a $\mathbb{P}^{n-1}$-bundle over $B$, the projection $p: X \rightarrow B$ 


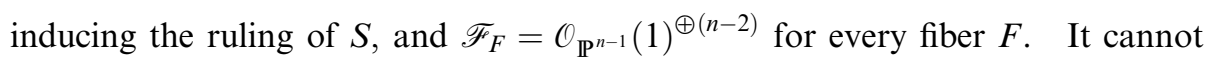
be $r<n-2$, since $\left(S, H_{S}\right)$ is not a scroll. Therefore $r=n-2$, and then $H_{F}=$ $\mathcal{O}_{\mathbb{P}^{n-1}}(2)$, since $H_{S} f=\left(2 C_{0}+f\right) f=2$. The converse is obvious and this concludes the proof.

Remark 8. Proposition 6 is effective, since $(S, L)$ is a very well known elliptic conic bundle in $\mathbb{P}^{5}$. We want to stress that Proposition 7 is effective as well. Arguing as in [26, Section 3] we can produce an example. Let $\mathscr{V}_{n}$ be an indecomposable vector bundle of rank $n$ and degree 1 over the elliptic curve $B$, and set $X:=\mathbb{P}\left(\mathscr{V}_{n}\right)$. We note that any two such bundles $\mathscr{V}_{n}, \mathscr{V}_{n}^{\prime}$ are related by $\mathscr{V}_{n}=\mathscr{V}_{n}^{\prime} \otimes \tau$, where $\tau$ is a line bundle of degree 0 on $B$. Thus $X$ is the same for all choices of $\mathscr{V}_{n}$. We also note that any such vector bundle $\mathscr{V}_{n}$ can be constructed inductively from a non-split exact sequence $0 \rightarrow$ $\mathcal{O}_{B} \rightarrow \mathscr{V}_{n} \rightarrow \mathscr{V}_{n-1} \rightarrow 0$, starting from a line bundle $\mathscr{V}_{1}$ of degree 1 . We have $h^{0}\left(\mathscr{V}_{n}\right)=1$ for all $n \geq 1$, hence the tautological line bundle $\xi$ on $X$ has a single section (up to a nonzero constant factor). Since the section of $\mathscr{V}_{n}$ vanishes nowhere on $B$, it follows that the corresponding section of $\xi$ vanishes exactly on $\mathbb{P}\left(\mathscr{V}_{n-1}\right)$. Note also that $\mathscr{V}_{n}$ is ample for any $n \geq 1$. Hence $\xi$ is ample. Now let $\xi_{1}, \ldots, \xi_{n-2}$ be $\xi$ twisted by the pullbacks on $X$ of $n-2$ distinct degree 0 line bundles on $B$ and let $\mathscr{E}=\bigoplus_{i=1}^{n-2} \xi_{i}$. Then $\mathscr{E}$ is an ample vector bundle on $X$. Consider its section $s=\left(s_{1}, \ldots, s_{n-2}\right)$ where $\left\langle s_{i}\right\rangle=H^{0}\left(\xi_{i}\right)$ and let $Z$ be its zero locus. Then $Z \cong \mathbb{P}\left(\mathscr{V}_{2}\right)$ [26, Claim B], i.e., $Z$ is the $\mathbb{P}^{1}$ bundle of invariant -1 over $B$, and $\xi_{Z}=\left[C_{0}\right], C_{0}$ being the tautological section. Now, letting $H:=2 \xi+F$ we have that $H$ is an ample line bundle, since $\xi$ is ample and $F$ is nef; Moreover $H_{Z}=\left[2 C_{0}+f\right]$ is very ample, due to Reider's theorem [31, Theorem 1].

Assuming that $S$ is not a ruled surface, assumption (VA) allows us to improve Proposition 5, probably roughly, as follows.

Corollary 6. Let $(X, \mathscr{E}, H)$ be as in $(\diamond)$ and suppose that (VA) holds. If $S$ is not a ruled surface, then $\delta \geq 2 g+11$.

Proof. Since $S$ is not ruled, (VA) implies $g \geq 3$, as the Enriques ruledness criterion, combined with the genus formula, immediately shows. Assume that $\delta \leq 2 g+10$. Then by Theorem 5 we see that $d \leq 10$. Furthermore, we have also

$$
2 g+10 \geq \delta=m_{2}-d=e(S)+4(g-1) \geq 4(g-1),
$$

i.e. $g \leq 7$. This allows us to use [27, Table in (4.0)]. We can write

$$
\delta=2 g+(e(S)+2 g-4)=2 g+D
$$


where $D:=e(S)+2 g-4=12 \chi\left(\mathcal{O}_{S}\right)-K_{S}^{2}+2 g-4$ by Noether's formula. Table in [27, (4.0)] shows that $S$ is birational to a K3 surface for $3 \leq g \leq$ 5 , but in this case $D \geq 24-K_{S}^{2}+2 \geq 26$, a contradiction. On the other hand, if $g=6$ and $S$ is of general type, then $D=63$, while in the remaining cases $D \geq 12-K_{S}^{2}+8 \geq 20$, except when $S$ is either an abelian or a bielliptic surface (Cases 8) and 9) in the Table), but in these two cases $S$ is minimal and this possibility is ruled out by [17, Theorem]. Finally, for $g=7$ condition $d \leq 10$ prevents $S$ from being birational to an abelian or a bielliptic surface (Cases 23) and 25) in the Table) and in the remaining cases we have $D \geq 12-K_{S}^{2}+10 \geq$ 21 , a contradiction.

\section{Acknowledgement}

The authors are grateful to Professor Y. Fukuma for calling [10] to their attention. They are also grateful to the referee for pointing out some gaps in the earlier version of the paper.

\section{References}

[1] M. Andreatta, E. Ballico, J. Wiśniewski, Vector bundles and adjunction, Intern. J. Math. 3 (1992), 331-340.

[2] W. Barth, Ch. Peters, A. Van de Ven, Compact Complex Surfaces, Springer, Berlin-New York, 1984.

[3] A. Beauville, Surfaces algébriques complexes, Astérisque, 54. Société Mathématique de France, Paris, 1978.

[4] M. Beltrametti, A. Lanteri, M. Palleschi, Algebraic surfaces containing an ample divisor of arithmetic genus two, Ark. Mat. 25 (1987), 189-210.

[5] T. de Fernex, Ample vector bundles with sections vanishing along conic fibrations over curves, Collect. Math. 49 (1998), 67-79.

[6] T. Fujita, Classification of polarized manifolds of sectional genus two, in Algebraic Geometry and Commutative Algebra. In honor of M. Nagata, 37-98, Kinokuniya, 1987.

[7] T. Fujita, Ample vector bundles of small $c_{1}$-sectional genera, J. Math. Kyoto Univ. 29 (1989), 1-16.

[8] T. Fujita, Classification Theories of Polarized Varieties, London Math. Soc. Lectures Notes Ser., vol. 155, Cambridge Univ. Press, 1990.

[9] T. Fujita, On certain polarized elliptic surfaces, in Geometry of Complex Projective Varieties (Cetraro, 1990), 153-163, Seminars and Conferences, vol. 9, Mediterranean Press, 1993.

[10] Y. Fukuma, A lower bound for sectional genus of quasi-polarized manifolds, J. Math. Soc. Japan 49 (1997), 339-362.

[11] Y. Fukuma, A note on a result of Lanteri about the class of polarized surfaces, Hiroshima Math. J. 46 (2016), 79-85.

[12] D. Fusi, A. Lanteri, Ample vector bundles with small $g-q$, Comm. Algebra 34 (2006), 2989-3008. 
[13] D. Gallarati, Una proprietà caratteristica delle rigate algebriche, Atti Accad. Naz. Lincei Rend. Cl. Sci. Fis. Mat. Nat. (8) 21 (1956), 55-56.

[14] D. Gallarati, Ancora sulla differenza tra la classe e l'ordine di una superficie algebrica, Ricerche Mat. 6 (1957), 111-124.

[15] R. Hartshorne, Algebraic Geometry, Springer-Verlag, New York-Heidelberg-Berlin, 1977.

[16] S. L. Kleiman, Tangency and Duality, in Vancouver Conference in Algebraic Geometry, Canad. Math. Soc. Conf. Proc. 6 (1986), 163-225.

[17] A. Lanteri, Ample vector bundles with sections vanishing on surfaces of Kodaira dimension zero, Matematiche (Catania) 51 (1996), suppl., 115-125 (1997).

[18] A. Lanteri, E. L. Livorni, Complex surfaces polarized by an ample and spanned line bundle of genus three, Geom. Dedicata 31 (1989), 267-289.

[19] A. Lanteri, H. Maeda, Ample vector bundles with sections vanishing on projective spaces or quadrics, Internat. J. Math. 6 (1995), 587-600.

[20] A. Lanteri, H. Maeda, Geometrically ruled surfaces as zero loci of ample vector bundles, Forum Math. 9 (1997), 1-15.

[21] A. Lanteri, H. Maeda, Special varieties in adjunction theory and ample vector bundles, Math. Proc. Cambridge Philos. Soc. 130 (2001), 61-75.

[22] A. Lanteri, H. Maeda, Elliptic surfaces and ample vector bundles, Pacific J. Math. 200 (2001), 147-157.

[23] A. Lanteri, M. Palleschi, Adjunction properties of polarized surfaces via Reider's method, Math. Scand. 65 (1989), 175-188.

[24] A. Lanteri, M. Palleschi, Complex projective surfaces of non-negative Kodaira dimension polarized by an ample and spanned line bundle of genus four, Indiana Univ. Math. J. 39 (1990), 85-104.

[25] A. Lanteri, M. Palleschi, A. J. Sommese, On the discriminant locus of an ample and spanned line bundle, J. reine angew. Math. 477 (1996), 199-219.

[26] A. Lanteri, A. J. Sommese, Ample vector bundles with zero loci having a hyperelliptic curve section, Forum Math. 15 (2003), 523-542.

[27] E. L. Livorni, On the existence of some surfaces, Algebraic geometry (L'Aquila, 1988), Lecture Notes in Math. 1417, Springer, Berlin, 1990, 155-179.

[28] H. Maeda, Nefness of adjoint bundles for ample vector bundles, Matematiche (Catania) 50 (1995), 73-82.

[29] E. Marchionna, Sopra una disuguaglanza fra i caratteri proiettivi di una superficie algebrica, Boll. Un. Mat. Ital. (3) 10 (1955), 478-480.

[30] M. Palleschi, C. Turrini, On polarized surfaces with a small generalized class, Extracta Math. 13 (1998), 371-381.

[31] I. Reider, Vector bundles of rank 2 and linear systems on algebraic surfaces, Ann. of Math. 127 (1988), 309-316.

[32] F. Serrano, Elliptic surfaces with an ample divisor of genus two, Pacific J. Math. 152 (1992), 187-199.

[33] F. Serrano, Fibrations on algebraic surfaces, in Geometry of Complex Projective Varieties (Cetraro, 1990), 289-301, Seminars and Conferences, vol. 9, Mediterranean Press, 1993.

[34] C. Turrini, E. Verderio, Projective surfaces of small class, Geom. Dedicata 47 (1993), $1-14$.

[35] K. Yokoyama, On blowing-up of polarized surfaces, J. Math. Soc. Japan 51 (1999), 523-533. 
Antonio Lanteri

Dipartimento di Matematica "F. Enriques"

Università degli Studi di Milano

Via C. Saldini, 50

I-20133 Milano, Italy

E-mail: antonio.lanteri@unimi.it

URL: http://users.mat.unimi.it/users/lanteri

Andrea Luigi Tironi

Departamento de Matemática

Universidad de Concepción

Casilla 160-C, Concepción, Chile

E-mail: atironi@udec.cl 